\title{
Rhythmic Whisking by Rat: Retraction as Well as Protraction of the Vibrissae Is Under Active Muscular Control
}

\author{
RUNE W. BERG ${ }^{1}$ AND DAVID KLEINFELD ${ }^{1,2}$ \\ ${ }^{1}$ Department of Physics and ${ }^{2}$ Neurosciences Graduate Program, University of California at San Diego, \\ La Jolla, California 92093
}

Submitted 24 July 2002; accepted in final form 24 September 2002

Berg, Rune W. and David Kleinfeld. Rhythmic whisking by rat: retraction as well as protraction of the vibrissae is under active muscular control. J Neurophysiol 89: 104-117, 2003. 10.1152/jn.00600.2002. The rhythmic motor activity of the vibrissae that rodents use for the tactile localization of objects provides a model system for understanding patterned motor activity in mammals. The muscles that drive this whisking are only partially fixed relative to bony attachments and thus shift their position along with the movement. As a means to characterize the pattern of muscular dynamics during different patterns of whisking, we recorded electromyogram (EMG) activity from the muscles that propel individual follicles, as well as EMG activity from a muscle group that moves the mystacial pad. The dominant pattern of whisking in our behavioral paradigm, referred to as exploratory whisking, consisted of large amplitude sweeps in the frequency range of 5-15 Hz. The frequency remained remarkably constant within a bout of whisking but changed values between bouts. The extrinsic musculature, which shifts the surface of the pad backwards, was found to be activated in approximate antiphase to that of the intrinsic muscles, which rotate individual vibrissae forward. Thus retraction of the vibrissae was driven by a backward shift in the attachment point of the follicles to the mystacial pad. In a less frequent pattern of whisking, referred to as foveal whisking, the vibrissae are thrust forward and palpate objects with low-amplitude movements that are in the higher frequency range of $15-25 \mathrm{~Hz}$. Protraction of the vibrissae remains driven by the intrinsic muscles, while retraction in this pattern is largely passive. Interestingly, a mechanical argument suggests that activation of the extrinsic muscles during foveal whisking is not expected to affect the angle of the vibrissae. As a means to establish if the phasic control of the intrinsic versus extrinsic muscles depended on sensory feedback, we characterized whisking before and after bilateral transections of the infraorbital branch of the trigeminal sensory nerve. The loss of sensory feedback had no net effect on the antiphase relation between activation of the intrinsic versus extrinsic muscles over the full frequency range for exploratory whisking. These data point to the existence of a dual-phase central pattern generator that drives the vibrissae.

\section{N T R O D U C T I O N}

Most processes of sensation involve the active repositioning of the underlying sensors. Therefore an understanding of sensation involves the need to decode the motor control of the sensory organs as well as the sensory input per se. At the level of vision, animals utilize smooth tracking movements as well as small saccadic movements to maintain the image of the selected object on the fovea (Rashbass 1961). Olfaction pro-

Address for reprint requests: D. Kleinfeld, Dept. of Physics 0319, Univ. of California, 9500 Gilman Dr., La Jolla, CA 92093 (E-mail: dk@physics.ucsd.edu). vides a second example of the motor control of a sensory process. Crustasea are observed to direct and flick their antennae as a means to detect and pursue the spatial distribution of attractants (Koehl et al. 2001), while mammals orient and increase their rate of sniffing in the presence of appetitive odorants (Freeman and Baird 1987). Somatosensation, for which sensory input is directly linked to the relative motion between the sensor and the object, provides evidence for the motor control of sensors as the substrate for texture analysis (Ahissar 1998; Darian-Smith 1984). The vibrissa system of rodents is unique among somatosensory systems in that muscular control has extensive bilateral mechanical symmetry (Brecht et al. 1997; Carvell and Simons 1990; Guic-Robles et al., 1989; Vincent 1912; Welker 1964; Wineski 1983), few degrees of freedom (Fee et al. 1997; Sachdev et al. 2002), and operates without spindle fibers to provide feedback on muscular contraction (Rice et al. 1994).

Here we address the muscular control of the macrovibrissae during rhythmic whisking by rat. These vibrissae are long, tactile hairs that originate from follicles that are arranged as an ordered array within a specialized facial structure, the mystacial pad (Dorfl 1982) (Fig. 1A). The rat uses its vibrissae to acquire tactile sensory information by sweeping them in a coordinated, rhythmic fashion (Brecht et al. 1997; Carvell and Simons 1990, 1995; Fee et al. 1997; Guic-Robles et al. 1989; Sachdev et al. 2002; Simons and Carvell 1996; Vincent 1912; Welker 1964; Wineski 1983). Whisking is behaviorally rich in that the animal can alter the amplitude and frequency between bouts (Nicolelis et al. 1995; O'Connor et al. 2002; Simons and Carvell 1996). Movement of the follicle is controlled by the facial motor nerve ("mn" in Fig. 1A), which innervates two classes of muscles. One class, the intrinsic muscles ("i" in Fig. 1B) (Dorfl 1982; Wineski 1985), have their points of attachment completely within the mystacial pad and form a sling around each follicle ("i" in Fig. 1B). Contraction of the intrinsic muscles has been shown to correlate with protraction of the vibrissae in a manner consistent with the force diagram of Fig. $1 D$ (Carvell et al. 1991). A second class of muscles, the extrinsic muscles, forms bridges from the surface of the pad ("e $\mathrm{e}_{\mathrm{u}}$ " in Fig. $1 B$ ) to anchors that lie external to the mystacial pad (" $\mathrm{e}_{\mathrm{u}}$ " and " $\mathrm{e}_{1}$ " in Fig. 1C). Contraction of the extrinsic muscles should shift the position of orifice of the follicle

\footnotetext{
The costs of publication of this article were defrayed in part by the payment of page charges. The article must therefore be hereby marked "advertisement" in accordance with 18 U.S.C. Section 1734 solely to indicate this fact.
} 
A
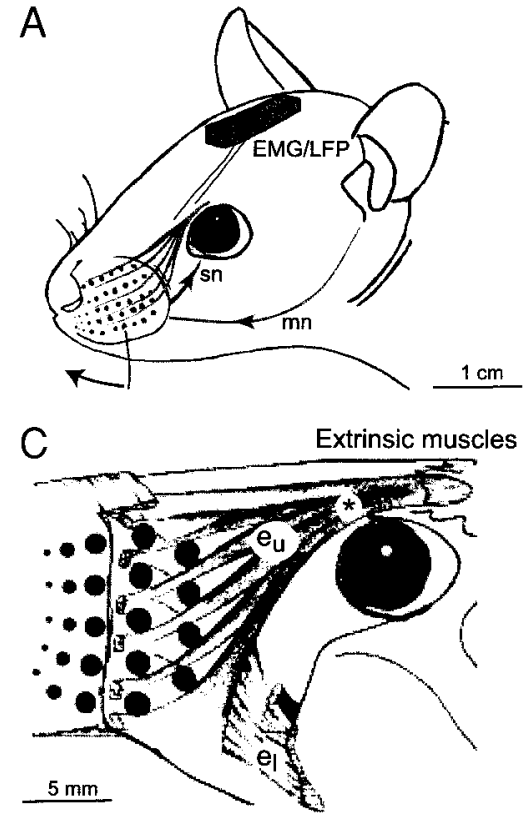

B
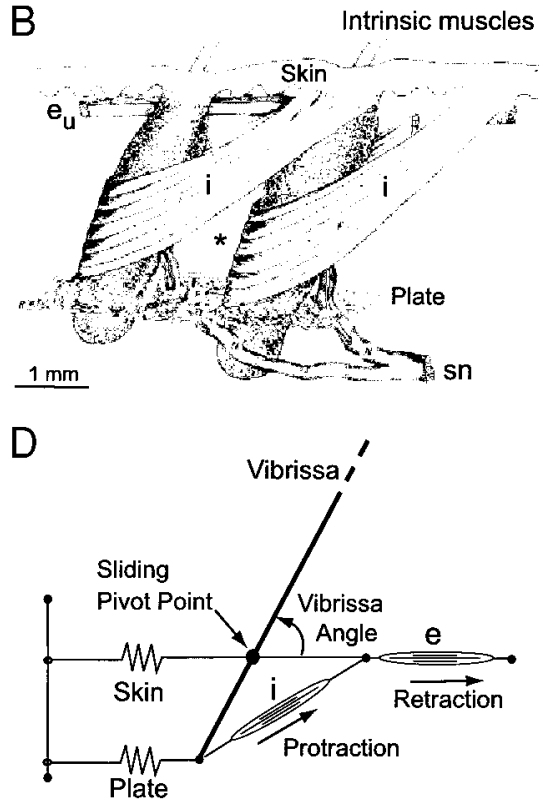

FIG. 1. Overview of the musculature of the vibrissae. The orientation in all panels is rostral to the left and caudal to the right. $A$ : drawing that indicates the location of the mystacial pad, the trigeminal (sensory) nerve (sn) and the facial (motor) nerve (mn). The EMG and LFP electrodes exit through the cap on the head of the animal. $B$ : anatomy of vibrissa follicles. The intrinsic muscle (i) forms a sling around the follicle to pivot the vibrissa forward. The trigeminal (sensory) nerve (sn) exits from the follicle (sn). The upper extrinsic muscle $\left(\mathrm{e}_{\mathrm{u}}\right)$ is anchored to the skin. The elastic connective tissue, shown as a fibrous sheet at the roots of the follicles, provides passive retraction when the vibrissa is pivoted forward. The approximate recording site of the intrinsic muscles of this study is indicated by an asterisk. $C$ : organization of the extrinsic musculature. The extrinsic muscles consist of 2 branches: An upper branch $\left(\mathrm{e}_{\mathrm{u}}\right)$ that includes the M. levator labii superioris and M. nasolabialis and a lower branch ( $\left.\mathrm{e}_{1}\right)$, the M. maxillo labialis. The black dots represent the follicles with the whisker coming out of the page. The recording site of the muscles of this study is indicated by an asterisk. $D$ : a model of the whisking mechanics, based on an interpretation of the anatomy of $B$ and $C$. Contraction of the intrinsic muscle (i) produces a torque that protracts the vibrissae, while contraction of the extrinsic muscle (e) moves the attachment point of the follicle and leads to retraction. The 2 springs in the model represent the elastic properties of the skin (top spring) and the fibrous connective tissue (bottom spring). Both springs are over-damped and are freely anchored in the vertical direction. The drawings in $B$ and $C$ were adapted from Figs. 1 and 3 in Dorfl (1982).

relative to the underlying plate and thus provide a force that shifts the pivot points of the vibrissae (Fig. 1D). This leads to the hypothesis that activation of the extrinsic muscles can drive retraction of the vibrissae (Wineski 1985). This hypothesis is supported by the observation that microstimulation of vibrissa primary motor cortex in anesthetized animals leads mainly to retraction of the vibrissae, as opposed to protraction (Gioanni and Lamarche 1985; Sanderson et al. 1984). It can be directly tested by simultaneously recording from both the intrinsic and extrinsic muscles.

We asked the following questions: 1) Are the extrinsic muscles, as opposed to only the intrinsic muscle, directly involved in rhythmic whisking? In particular, we test the hypothesis that retraction of the vibrissae can be driven by the extrinsic muscles. 2) What is the spectral fidelity of rhythmic whisking? In particular, how does the variability of rhythmic whisking within a bout of whisking compare with the variability between different bouts of whisking? 3) What is the detailed role of sensory feedback in the control of rhythmic whisking? In particular, we quantify the spectral properties of rhythmic whisking, and the phased activation of different muscle groups, before and after transection of the sensory nerve.

Our experiments made use of a defined behavior task in which animals perch and whisk in air in search of a food tube. The electromyogram (EMG) of the intrinsic muscles and of the upper branch of the extrinsic muscles were concurrently measured. The relation between EMG activity and physical motion of the vibrissae was established with videography. As whisking is naturally a rhythmic process, we made extensive use of spectral techniques and associated statistical measures to characterize the muscular activation.

Preliminary aspects of this work have been presented (Berg and Kleinfeld 2001).

\section{METHODS}

Our subjects were nine Long-Evans female rats, 200-300 g in mass, that were trained to whisk in search of a food reward. The differential electromyogram ( $\nabla \mathrm{EMG})$ of the intrinsic muscles and the $\nabla \mathrm{EMG}$ of the upper branch of the extrinsic muscles was concurrently measured in eight of the nine animals. The $\nabla$ EMG was calculated as the difference in the potential measured by electrodes places across the muscle group. We further recorded the differential local field potential $(\nabla$ LFP) from vibrissa primary sensory (S1) cortex in each of these animals, as previously described (O'Connor et al. 2002). After a set of $\nabla$ EMG and $\nabla$ LFP data were obtained, we performed a bilateral transection of the infraorbital branch of the trigeminal nerve (IoN) on five of the eight animals with EMG electrodes in both the intrinsic and extrinsic muscles. Sham transection surgery was performed on two of the remaining three animals as a control. This progression is summarized as:

Training $\rightarrow$ EMG surgery $\rightarrow$ recording $\rightarrow$ bilateral IoN transection $\rightarrow$ recording

Last, the $\nabla$ EMG of the intrinsic muscles only was recorded in the remaining original animal. The care and all aspects of experimental manipulation of our animals were in strict accord with guidelines from 
the National Institute of Health (1985) and have been reviewed, approved, and observed by members of the local Institutional Animal Care and Use Committee.

\section{Behavioral training}

The rats were gentled and acclimatized to the experimental environment over a period of 1-2 wk prior to surgical implanting of the EMG and LFP electrodes. After a 10-day recovery from surgery, rats were deprived of solid food and trained to explore a figure eight maze as a means to gain access to liquid food [50\% (wt/vol); LD-100; PMI Feeds; Fig. 2]. Small objects were occasionally introduced to the maze to encourage exploratory whisking. Food was presented on an episodic basis through two venues. The first food presentation venue was through a mechanized food tube, located below a video camera (see Videography), that can swing into place. The geometry of this set-up forced the animals to perch at a ledge and crane to gain access to the food tube (Fig. 2). The second venue was through a hand-held syringe that was placed at different locations. Each recording session lasted about $1 \mathrm{~h}$, and a total of $10 \mathrm{ml}$ of liquid food was typically imbibed in a session. Recording was repeated daily for 3-7 days.

The viability of our animals was assayed, in part, by the spectral composition of the VLFP (O'Connor et al. 2002). We collected data only during intervals in which we observed a relatively broad spectral response in cortex that was centered near $7 \mathrm{~Hz}$. This response is consistent with the largely desynchronized electrical state of cortex in an animal during exploratory behavior (O'Connor et al. 2002; Semba and Komisaruk 1984). Additional intervals, in which the rats were immobile and the vibrissae exhibited low-amplitude tremors, were characterized by a spectral response that was sharply peaked between 9 and $11 \mathrm{~Hz}$. These intervals correspond to a thalamocortical spindling (Buzsaki et al., 1988; Semba and Komisaruk 1984) and were rejected for further analysis.

\section{$E M G$}

Microwire EMG electrodes fashioned from Teflon-coated Tungsten wire (50 $\mu \mathrm{m}$; no. 7955, A-M Systems) were surgically implanted as a

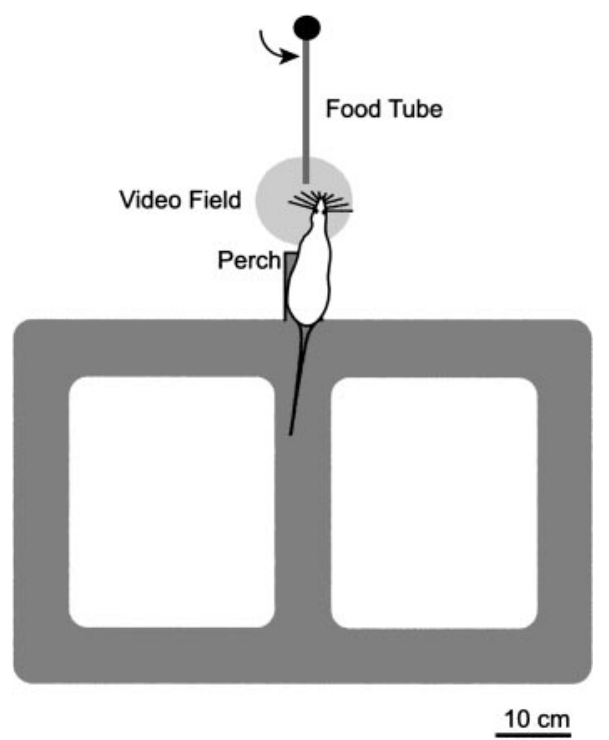

FIG. 2. Schematic of the maze used to train the rats and observe their whisking. Animals were free to walk on the figure 8 platform. Aliquots of liquid food were presented through a rotatable tube that was located opposite a perch. The animals had to crane to locate and reach the tube. A high-speed video camera could record vibrissa motion in the area that included the perch and the food tube; a pair of stroboscopic light emitting diodes provided oblique illumination of the head of the animals. means to record extracellular muscle activity. All procedures were performed on animals that were anesthetized by a mixture of ketamine $(0.05$ $\mathrm{mg} / \mathrm{g}$ rat mass, supplemented every $2 \mathrm{~h}$ at $0.01 \mathrm{mg} / \mathrm{g}$ rat mass) and xylazine $(0.015 \mathrm{mg} / \mathrm{g}$ rat mass) that was delivered intraperitoneally.

\section{Intrinsic muscles}

Microwires were placed within the mystacial pad from underneath the skin, as previously described (Carvell et al. 1991; Fee et al. 1997), as a means to record the aggregate activity of the intrinsic muscles (Fig. $1 B$ ). In brief, an incision was made through the skin along the midline of the skull. A 25-gauge syringe needle was loaded with a set of four electrodes and inserted below the incised skin and through the soft muscular tissue of the mystacial pad. The needle exited through the rostral end of the pad, the tips of the wires were stripped of insulation to form EMG electrodes, and individual wires were pulled back so that the set of wires were spaced uniformly along the pad (e.g., location * in Fig. 1B).

\section{Extrinsic muscles}

Microwires were placed in the fibers of the upper extrinsic musculature, the M. levator labii superioris (Dorfl 1982) and M. nasolabialis (Dorfl 1985; Wineski 1985), as a means to record the aggregate activity of part of the musculature that moves the mystacial pad (Fig. $1 C$ ). This group of muscles is accessed through the incision along the midline of the skull. These muscle groups attach on the frontal bone behind the nasofrontal suture, close to the incision. They were identified during surgery by applying a small oscillatory current with a bipolar electrode and by observing if the vibrissae moved in backwards direction. Four fine wires were gently pressed into the fibers on each side and sutured to the connective tissue with 4-0 nylon suture (e.g., location $*$ in Fig. 1C). The electrical reference for both the intrinsic and extrinsic extracellular signals was placed in the dermis that lay dorsal to the nasal bone.

\section{Verification of signals}

After completion of all behavioral measurements, the position of both sets of EMG microwire electrodes was confirmed in selected animals. We passed trains of monophasic, bipolar current-pulses, 200 $\mu \mathrm{s}$ in duration repeated at an interval of $1 \mathrm{~ms}$ for a total of five pulses per train and 100-200 $\mu \mathrm{A}$ in amplitude through pairs of wires in each set. The concomitant movement of the vibrissae elicited by these pulses was measured in two ways. For all animals, we glued a small magnet, $<1 \mathrm{mg}$ in mass, to vibrissa $\mathrm{C} 2$, and recorded the direction and extent of deflection through a magnetoresistive detector (HMC1001; Honeywell, Minneapolis, MN; Fig. $3 A$ and $B$ ). In selected animals, the motion of the vibrissa was further confirmed with high-speed videography (Figs. 3, $\mathrm{C}-\mathrm{H}$ ).

\section{Bilateral transection of the infraorbital nerve}

In most subjects, the IoN was transected after a 3- to 7-day period of data collection with the animals in the normal state. The nerves were cut from inside the orbit to avoid interference with the facial motor nerve (VII cranial nerve) and to avoid disturbing the EMG electrodes. In brief, the eye was retracted in the caudal direction, the tissue anterior to the eye was split, and the nerve was identified along the dorsal ridge of the orbit. All branches of the IoN, reported to number eight (Dorfl 1985), were transected. After recovery, the animal lacked the behavioral correlates of facial sensation as visually assayed by the inability to cease whisking on contact with an object, but was able to locate and imbibe water and liquid food.

\section{Data acquisition}

All electrical signals were buffered near the head of the animal with field effect transistors (NB Laboratories, Denville, TX). The extracel- 
A

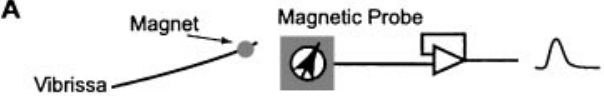

B Extrinsic Stimulation
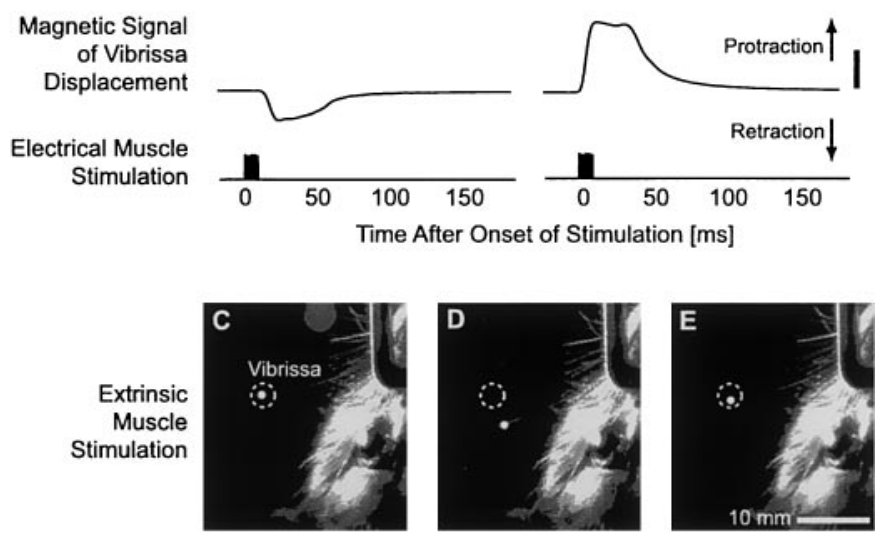

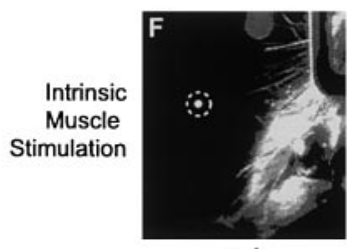

$t=0$

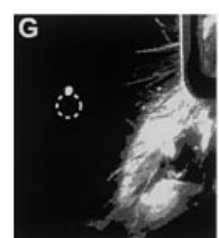

$\mathrm{t}=27 \mathrm{~ms}$

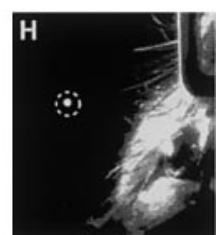

$\mathrm{t}=54 \mathrm{~ms}$
FIG. 3. Movement of the vibrissae in response to electrical stimulation of the microwire EMG electrodes. The animal was anesthetized on ketamine $(0.05 \mathrm{mg} / \mathrm{g}$ rat mass $)$ and Xylazine $(0.015 \mathrm{mg} / \mathrm{g}$ rat mass $)$. A: cartoon of apparatus to electrically measure the deflection of vibrissa C2. A small rare earth magnet is glued to the trimmed vibrissa. Movement is recorded either as a voltage that is proportional to the change in the magnetoresistance of the probe or, alternatively, by videography. $B$ : vibrissa deflection as a response to stimulation through the EMG wires of intrinsic and extrinsic muscles, respectively. The sign of the signal shows that activation of the extrinsic muscle leads solely to retraction while activation of the intrinsic muscle leads solely to protraction. Scale bar is $5^{\circ}$. $C-H$ : videographs of the vibrissa response to stimulation through the EMG wires of intrinsic and extrinsic muscles, respectively. A small drop of reflecting glue was placed on vibrissa $\mathrm{C} 2$ and back illumminated. Rostral is up and the broken circle is a fiducial and a bar was drawn along the vibrissa for clarity. Note again that activation of the extrinsic muscle leads to retraction while activation of the intrinsic muscle leads to protraction.

lular signals from the intrinsic and extrinsic muscles were differentially amplified $(\times 6,400)$ relative to the nasal reference and digitized at $8 \mathrm{kHz}$ with a 12-bit D/A converter (AT-MIO-16E-1, National Instruments). The rectified, differential EMG was formed numerically (Interactive Data Language; Research Systems). We first computed the difference of two signals that spanned a muscle group to remove common artifacts and form the $\nabla \mathrm{EMG}$, e.g., the voltage on the wire in the posterior end of the mystacial pad was subtracted from the voltage on the wire at the anterior end to form the intrinsic muscle signal. This difference signal was high pass filtered at $50 \mathrm{~Hz}$, the absolute value was formed, and the now rectified signal was low-pass filtered at $80 \mathrm{~Hz}$ and subsampled at $800 \mathrm{~Hz}$.

\section{Videography}

The motion of the vibrissa was directly measured by a high-speed videography (100-111 frames/s; model ES310 charge coupled device camera; Kodak) for selected trials. In these cases, the animals whisked as they perched on the maze and stretched toward the food tube. The vibrissae were illuminated under pseudo-darkfield conditions (Fee et al. 1997). Frame-by-frame reference signals from the video electronics were used to synchronize video frames with the digitized EMG signals. While the $\nabla \mathrm{EMG}$ provided a signal that was proportional to the depolarization of the underlying musculature, videography allowed us to directly estimate the angular position of the vibrissae over time.

\section{Spectral analysis}

Spectra power densities of individual time series of muscle or brain activity, denoted S(f) below, and the SD of these measures, were calculated with the direct multi-taper spectral estimation techniques of Thomson (1982) (see Cacciatore et al. (1999) for implementation). In brief, the spectral power is defined in terms of an average over all instances and tapers

$$
S(\mathrm{f}) \equiv \frac{1}{\mathrm{~N}} \frac{1}{\mathrm{~K}} \sum_{\mathrm{n}=1}^{\mathrm{N}} \sum_{\mathrm{k}=1}^{\mathrm{K}} \tilde{\mathbf{V}}^{(\mathrm{n}, \mathrm{k})} \tilde{\mathbf{V}}^{*(\mathrm{n}, \mathrm{k})}
$$

where $*$ means complex conjugation and

$$
\tilde{\mathbf{V}}^{(\mathrm{n}, \mathrm{k})} \equiv\left\{\tilde{\mathrm{V}}^{(\mathrm{n}, \mathrm{k})}(f)\right\}_{\mathrm{f}=0}^{\mathrm{f}_{\mathrm{N}}}=\sum_{\mathrm{t}=0}^{\mathrm{T}} \mathrm{e}^{\mathrm{i} 2 \pi \mathrm{ft}} w^{(\mathrm{k})}(t) \mathrm{V}^{(\mathrm{n})}(t) \cdot t_{\mathrm{S}}
$$

is the discrete Fourier transform of the product of the time series, $\mathbf{V}^{(\mathrm{n})} \equiv\left\{\tilde{\mathrm{V}}^{(\mathrm{n})}(t)\right\}_{\mathrm{t}=0}^{\mathrm{T}}$, times the $k$-th taper, $\mathbf{w}^{(\mathrm{k})} \equiv\left\{w^{(\mathrm{k})}(t)\right\}_{\mathrm{t}=0}^{\mathrm{T}}$ and the parameter $t_{\mathrm{S}}$ is the time per point of the subsampled data $(2 \mathrm{~ms}$ in the present work). Numerically, $\tilde{\mathbf{V}}^{(\mathrm{n}, \mathrm{k})}$ was computed as the fast Fourier transform of the product after it was padded to $\geq 4$ times the initial length. The parameter $N$ is the number of instances of the waveform $\left(1-10^{3}\right.$ in the present work), $K$ is the number of tapers or degrees of freedom in the spectral estimate (1-5 in the present work), $T$ is duration of the data trace (1-4 s in the present case), and $f_{\mathrm{N}}=\left(2 t_{\mathrm{S}}\right)^{-1}$ is the Nyquist frequency. In this procedure, the spectrum is averaged over a half-bandwidth of $(K+1) /(2 T)$.

A special aspect of this spectral estimation techniques is that it minimizes the leakage between neighboring frequency bands. Additional smoothing, but no change in bandwidth, is obtained by averaging the spectra from multiple instances. SD of the power spectra are reported as jackknife estimates across trials (Thomson and Chave 1991). Last, the spectral coherence and the variance in the coherence, used in this study to determine the phase lag between two signals, was similarly calculated with the techniques of Thomson (Thomson 1982; Thomson and Chave 1991).

\section{RES U L T S}

A necessary condition for the active retraction of the vibrissae is that electrical activation of the extrinsic muscles must cause a deflection in the caudal direction. Conversely, stimulation of the intrinsic muscles in the mystacial pad are expected to cause movement in the rostral direction, consistent with protraction. We confirmed these effects, first discussed by Wineski (1985), by passing current pulses through the microwires to the respective muscle groups and observing the resultant motion (Fig. 3). Stimulation of the extrinsic muscles led to prompt, caudal deflections as seen through the electrically measured deflection of a small magnet attached to vibrissa $\mathrm{C} 2$ (Fig. 3, $A$ and $B$ ) and through videography (Fig. 3, $C-E$ ). Conversely, stimulation of the intrinsic muscles led to prompt, rostral deflections (Fig. 3, $B$ and $F-H$ ). The difference in direction of vibrissa movement seen for activation of the extrinsic versus intrinsic muscles was observed in each of our animals $(n=9)$.

We now shift our focus to the whisking behavior of rats as they explored the maze in search of a food tube or food laden syringe. Our animals were observed to exhibit two patterns of rhythmic whisking. The most prevalent pattern consisted of 1 - to 10 -s bouts 

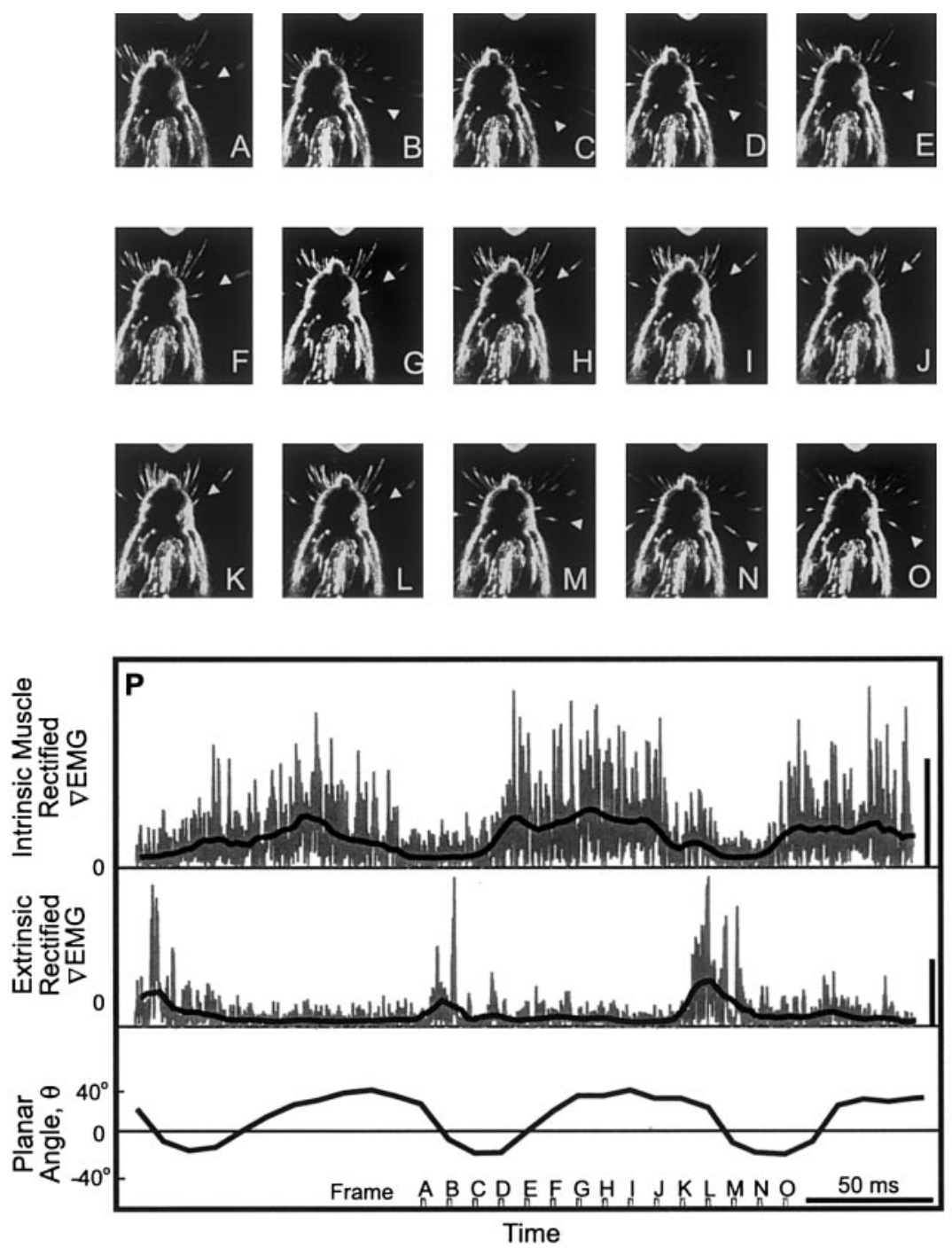

FIG. 4. Simultaneous videography and rectified $\nabla$ EMG activity during low frequency, exploratory whisking. $A-O$ : consecutive frames of whisking as the rat whisks freely in air. $P$ : rectified $\nabla$ EMG activity of both the intrinsic and extrinsic muscles on the right side during the whisking bout that encompassed the above videographs (top and middle). We further show (bottom) the angular position of the right vibrissae as determined from the videography with the angle defined as in Fig. $5 P$. The side bars represent $100 \mu \mathrm{V}$. $Q$ : $\nabla$ EMG activity of both the intrinsic and extrinsic muscles during low-frequency, exploratory whisking in a separate epoch. Inset: correlation coefficient between the $\nabla$ EMGs for the 2 muscle groups. Note the high coherence between the 2 muscle groups, which peaks at a phase lag of $0.9 \pi$ radians.

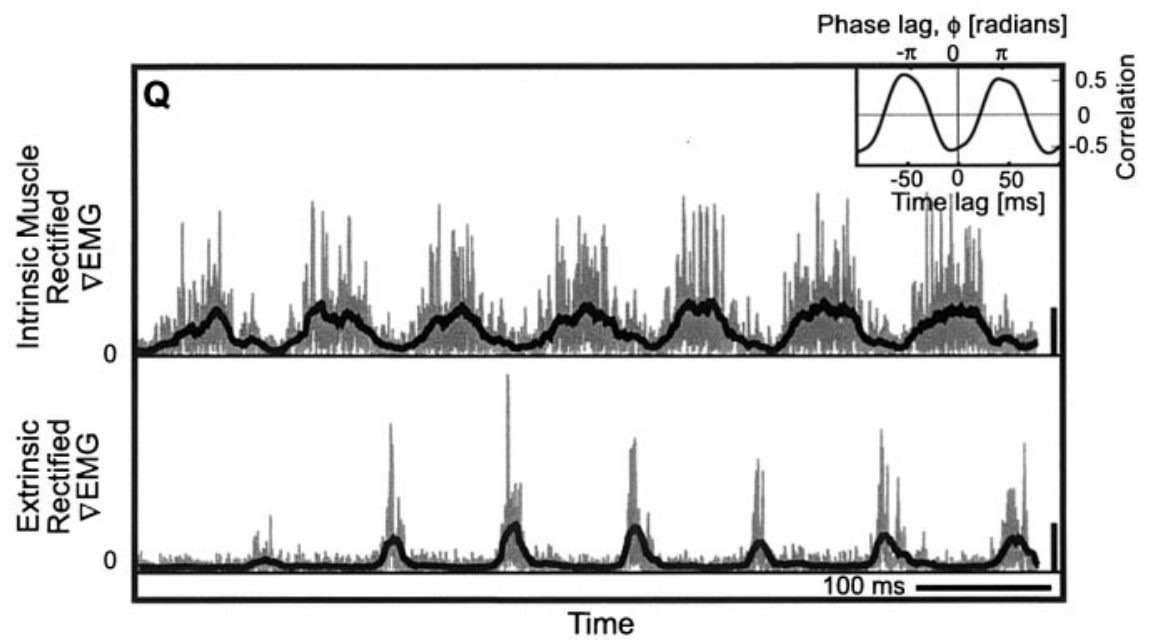

of large amplitude whisks, with a frequency in the range of 5-15 $\mathrm{Hz}$. We refer to this movement as "exploratory" whisking. The form of the motion is in agreement with past reports (Carvell and Simons 1990; Fee et al. 1997; O'Connor et al. 2002; Vincent 1912; Welker 1964). A second pattern of whisking was observed when animals had to crane across a gap to locate the food tube or other objects of interest. The animals thrust their vibrissae forward and rhythmically palpated the object with their vibrissae for periods of $0.5-1 \mathrm{~s}$. We refer to this pattern as "foveal" whisking since the vibrissae are clustered in front of the head in a relatively dense pattern, similar to the clustering of photoreceptors in the fovea of the retina. Compared with exploratory whisking, the 
motions in foveal whisking were of higher frequency, ranging from 15 to $25 \mathrm{~Hz}$, and of smaller amplitude. The observation of higher frequency whisking is also consistent with past observations (Carvell and Simons 1990, 1996).

The presence of two patterns of whisking in our behavioral paradigm allowed us to characterize muscular activation over a broad range of values of angular set points. We first report the relation between the angular motion of the vibrissae and the activation of intrinsic versus extrinsic muscles. These data are based on the comparison of a sequence of videograph images of the vibrissae with the rectified $\nabla \mathrm{EMG}$ signals (data from 4 animals). We then report the parameterization of the muscular activation and whisking dynamics in terms of the distribution of measured quantities across an extensive sample of whisking bouts and animals (data from 9 animals).

\section{Vibrissa motion during exploratory whisking}

Successive video images of a representative bout of exploratory whisking shows that the vibrissae are swept with large amplitude motions, at a rate of $9 \mathrm{~Hz}$, that span from highly retracted to highly protracted angles (Fig. 4, A-O). Protraction of the vibrissae follows activation of the intrinsic muscles, as inferred from the rectified intrinsic $\nabla \mathrm{EMG}$, while retraction of the vibrissae follows activation of the extrinsic muscles (Fig. $4 P$ ). The angle of the vibrissae (defined in Fig. 5P), was estimated from each image ( $\boldsymbol{\Delta}$ in Fig. $4, A-O$ ). The time delay between a change in angular position and the intrinsic $\nabla \mathrm{EMG}$ signal is $20 \mathrm{~ms}$ in this example (Fig. 4P).

The antiphasic activation of extrinsic versus intrinsic muscles is seen in further detail in a second example of exploratory whisking (Fig. 4Q). The cross-correlation between the $\nabla \mathrm{EMG}$ of the two muscle groups indicates that the phase lag between extrinsic and intrinsic muscle activity, denoted $\phi$, is $\phi=0.9 \cdot \pi$ radians (inset in Fig. $4 Q$; the lag is calculated with respect to the intrinsic $\nabla \mathrm{EMG})$. In general, the antiphasic activation of the two muscle groups (Fig. 4, $P$ and $Q$ ), together with the correspondence of retraction with the activation of the extrinsic muscles (Fig. 4P), was observed in all videographed sets of epochs $(n=20 ; 4$ animals). These results imply that retraction of the vibrissae during exploratory behavior is an active process.
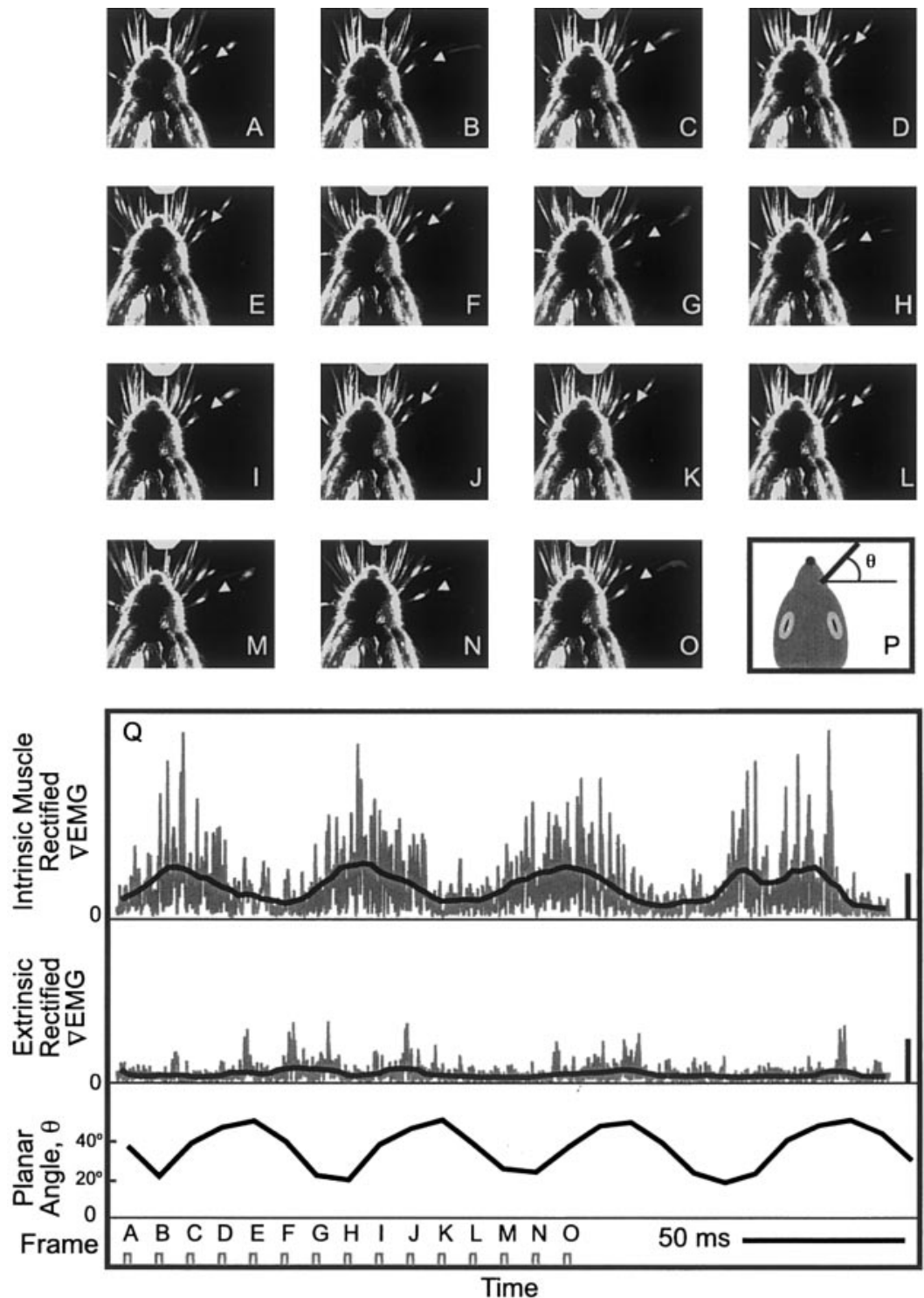

FIG. 5. Simultaneous videography and rectified $\nabla$ EMG activity during high-frequency, foveal whisking. $A-O$ : consecutive frames (2-ms exposure recorded at 10-ms intervals) of whisking as the vibrissae palpate a food tube during foveal whisking. The tube appears as a small bump at the top of each frame. $P$ : illustration of the planar angle, defined with respect to a normal to the body axis. $Q$ : rectified $\nabla$ EMG activity of both the intrinsic and extrinsic muscles on the right side during the whisking bout that encompassed the above videographs (top and middle). We further show (bottom) the angular position of the right vibrissae as determined from the videography ( $\triangle$ in $A-O$; the frames are indicated by pulses in the bottom row). Note that the rectified $\nabla$ EMG activity for the extrinsic muscles is essentially silent. The side bars represent $100 \mu \mathrm{V}$. 


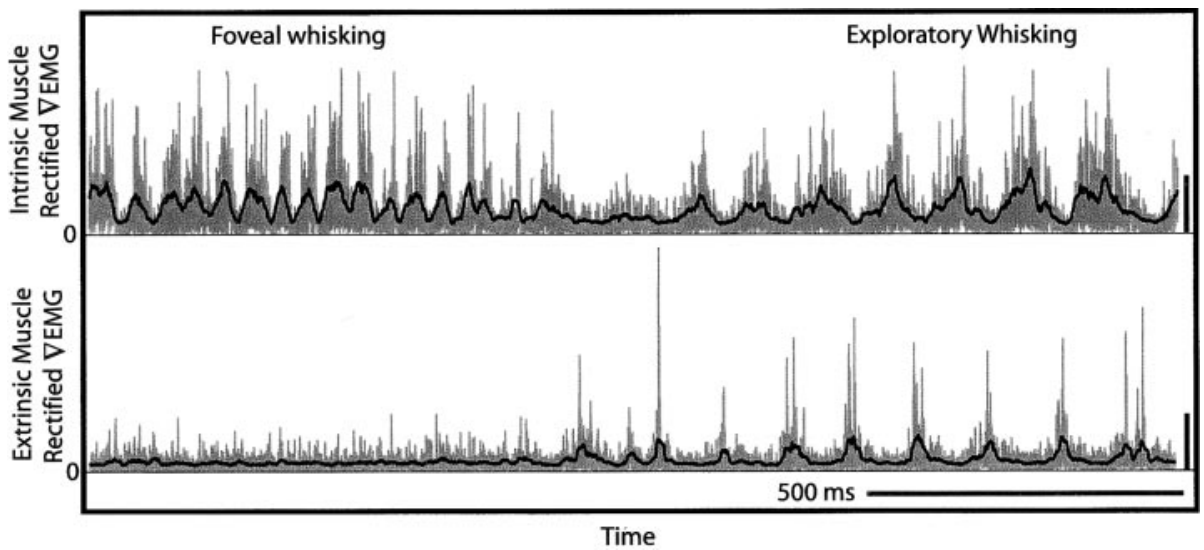

FIG. 6. Example of concurrent foveal and exploratory whisking to show the transition from foveal whisking, at $22 \mathrm{~Hz}$ in this example, to exploratory whisking, at $9 \mathrm{~Hz}$. The top trace is the rectified $\nabla \mathrm{EMG}$ of the intrinsic muscles; the black line is the smoothed data. The bottom trace is the $\nabla \mathrm{EMG}$ of the extrinsic muscles; the black line is the smoothed data. Note that the extrinsic muscles are inactive during the $22-\mathrm{Hz}$ whisking, but are rhythmically active during the $9-\mathrm{Hz}$ whisking. Scale bars indicate $100 \mu \mathrm{V}$.

\section{Vibrissa motion during foveal whisking}

In contrast to the case for exploratory whisking, a representative bout of foveal whisking shows that the vibrissae are largely thrust forward. The rhythmic motion is superimposed on this offset in angle (Fig. 5, $A-O$ ) and is smaller in amplitude and much more rapid, $17 \mathrm{~Hz}$ in this example, than in the case of exploratory whisking. The amplitude of the motion for the caudal vibrissae (Fig. 5P), which showed the largest motion, was estimated from each image ( $\boldsymbol{\Delta}$ in Fig. $5, A-O$ ). We found that the position is locked to the $\nabla \mathrm{EMG}$ signal for the intrinsic muscles; the extrinsic muscle is largely inactive (Fig. 5Q). Thus protraction of the vibrissae follows activation of the intrinsic muscles and retraction is provided by the vasoelastic properties of the muscle and tissue. The time delay between the

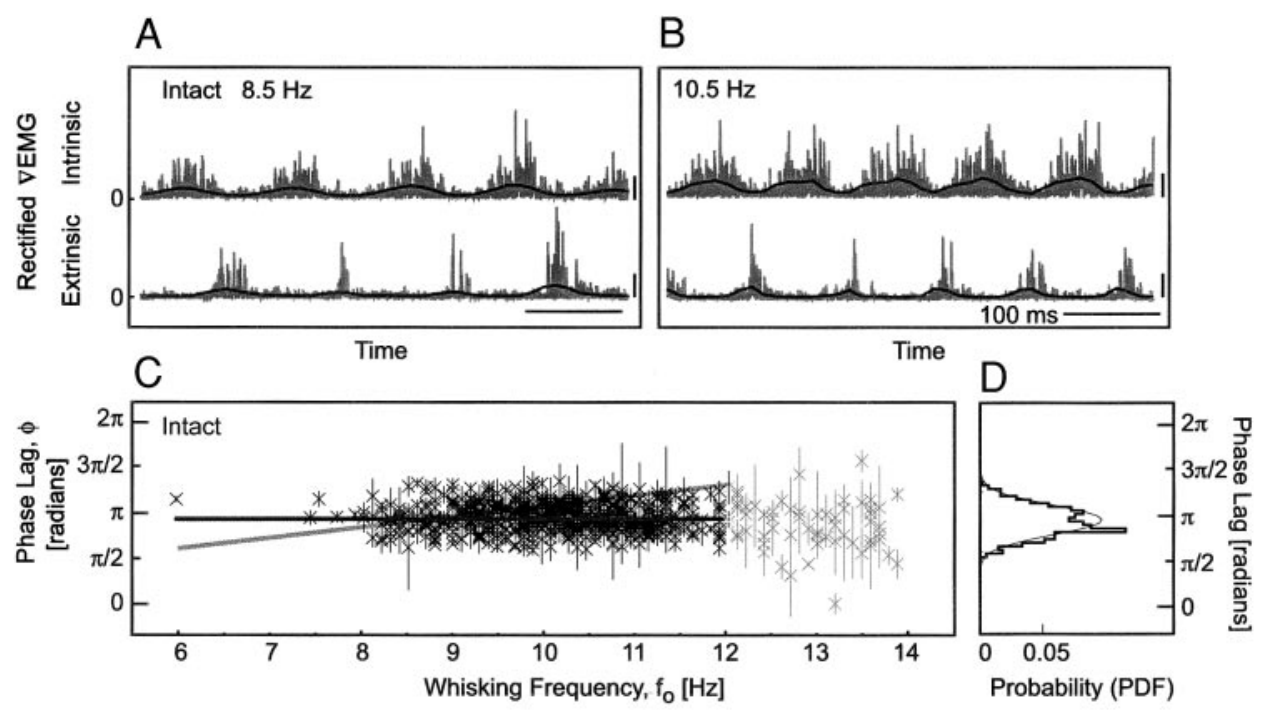

E

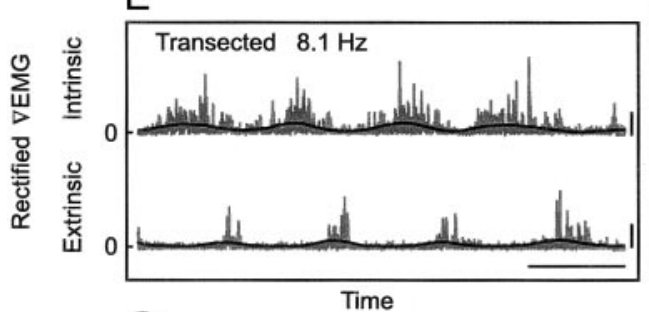

$\mathrm{F}$

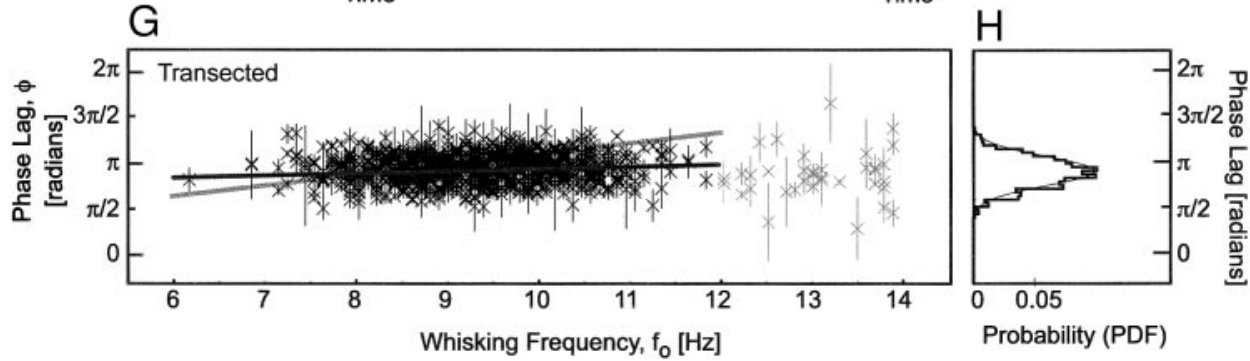

FIG. 7. The phase relation between rectified $\nabla$ EMG activity in the intrinsic vs. extrinsic muscles as a function of whisking frequency for a representative animal. $A$ and $B$ : examples of whisking at 2 frequencies, with the nervous system "intact," to illustrate that the relative phase between activation of the intrinsic vs. extrinsic muscles does not vary with frequency. $C$ : distribution of phase lags ( $n=603$ whisking bouts); a value $0<\phi<$ $\pi$ corresponds to the intrinsic muscles leading in time. The preponderance of the data points, $>90 \%$, lie between 6 and $12 \mathrm{~Hz}$. The dark gray line is a best fit to the data, with a slope of $-0.01 \pm 0.02$ radians $/ \mathrm{Hz}$. The light gray line is the expected phase shift for a time delay of $\tau=350 \mathrm{~ms}$, i.e., $\phi=2 \pi \tau$, for the activation of the extrinsic vs. intrinsic muscles. $D$ : PDF for the phase after integration over frequencies from 5 to $12 \mathrm{~Hz}$. The thin line is a best fit of a Gaussian distribution with a mean value of $\phi=1.0 \cdot \pi$ radians. The deviation of the data from a Gaussian fit is small but statistically significant. $E$ and $F$ : examples of whisking at 2 frequencies after transection of the IoN; as in $A$ and $B$, the relative phase between activation of the intrinsic vs. extrinsic muscles does not vary with frequency. $G$ : distribution of phases in the "transected" case $(n=9801$-s whisking bouts). As in the "intact" case, the preponderance of the data points lie between 6 and 12 $\mathrm{Hz}$. The dark gray line is a best fit to the data, with a slope of $0.05 \pm 0.02 \mathrm{radians} / \mathrm{Hz}$. $H$ : probability distribution function for the phase in the "transected" animal after integration over all frequencies. The distribution peaks at $\phi=1.0 \cdot \pi$ radians and is statistically indistinguishable from the case for the "intact" animal $(C)$. 


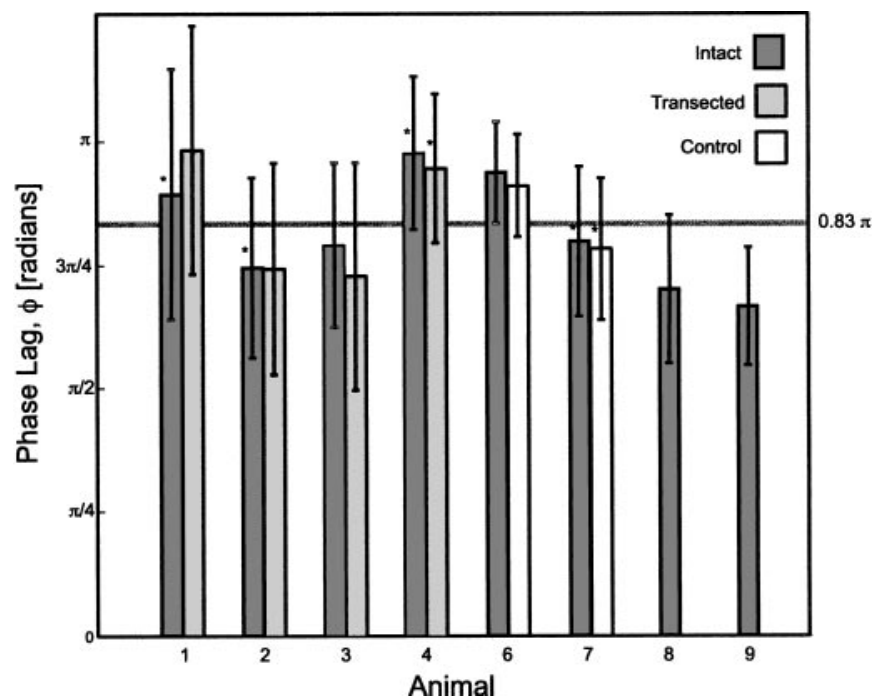

FIG. 8. Summary of the phase lag between the rectified $\nabla$ EMG activity in the intrinsic vs. extrinsic muscles for all animals. Each column represents the mean and SD for the distribution (PDF) of phase lags (Fig. 7, $D$ and $H$ ). The number of whisking bouts, denoted as intact/(transected or control), is 476/269, 509/364, 207/93, 603/980, 634/368, 659/407, 338/-, and 96/- for animals 1 through 4 and 6 through 9 , respectively. The data in the columns marked with an asterisk deviate from a Gaussian distribution (Fig. 7, $D$ and $H$ ). The gray line is the sample average.

onset of the two signals is $18 \mathrm{~ms}$ in this example, close to that for the case of foveal whisking.

The correspondence of retraction with the activation of the extrinsic muscles (Fig. 5Q) was observed in all videographed sets of epochs ( $n=20 ; 4$ animals). These results imply that retraction of the vibrissae during foveal whisking appears to be passive.

\section{Transition between patterns}

While exploratory whisking was the prevalent pattern observed in our behavioral paradigm, animals were observed to transition between the two modes as they craned to gain access to the food tube (Fig. 2). We consider a record with a progression from foveal to exploratory whisking (Fig. 6). During the foveal pattern, rhythmic activation of the intrinsic muscle but essentially no activation of the extrinsic muscles is present, as above (Fig. 5Q). The onset of exploratory whisking is accompanied by a decrease in whisking frequency from 22 to $9 \mathrm{~Hz}$ and by activation of both the intrinsic and extrinsic muscles in anti-phase, also as above (Fig. 4, $P$ and $Q$ ).

\section{Delay between the intrinsic $\nabla E M G$ and protraction}

The $\nabla \mathrm{EMG}$ is proportional to the force produced by the musculature. For small angular displacements, the $\nabla \mathrm{EMG}$ is further proportional to the torque applied to the vibrissae. We note from physical mechanics that dissipation by the viscoelastic properties of the mystacial pad will lead to a temporal lag between the displacement of the vibrissae and the intrinsic $\nabla \mathrm{EMG}$, as is evident in the examples of Figs. $4 P$ and $5 Q$ (see also Fig. $2 B$ in Carvell et al. 1991). We measured this lag, denoted $\tau_{\text {lag }}$, over a 6 - to $25-\mathrm{Hz}$ range of whisking frequencies ( $n=68$ cycles; 3 animals) and found that it was essentially independent of whisking frequency, with

$$
\tau_{\mathrm{lag}}=24 \pm 6 \mathrm{~ms}(\text { mean } \pm \mathrm{SD}) .
$$

The uncertainty is dominated by the frame rate of the videography.

\section{Phase between intrinsic and extrinsic muscle activity}

Our observations on bouts of exploratory whisking showed that there was a phase lag between activation of the intrinsic and extrinsic muscles (Figs. 6 and $4, P$ and $Q$ ). A central question in the interpretation of this result is whether the phase lag is independent of frequency, which implies that the trajectory of vibrissae motion during exploratory whisking is independent of frequency, or whether the lag varies with frequency, which is suggestive of a time delay between the activation of the two muscle groups.

The result for a representative animal shows that the phaselag remains fixed as the frequency of whisking changes from bout to bout (cf. Fig. 7, $A$ and $B$ ). To quantify the relative muscular activation during whisking, we calculated the phase lags between the intrinsic and extrinsic $\nabla \mathrm{EMG}$ across all whisking frequencies (Fig. 7C). The result for this animal $(n=$ 603,1 -s bouts) shows that the phase is constant over a 6- to $12-\mathrm{Hz}$ range of exploratory whisking for this animal (dark gray line in Fig. 7C); this range encompassed $90 \%$ of all whisking bouts. The average phase lag, found by integrating over the 6to $12-\mathrm{Hz}$ range of frequencies to form the PDF of the phase lag, has a value of nearly $\phi \approx \pi$ radians (Fig. $7 D$ ); the small fraction of phase lags for $f_{0}>12 \mathrm{~Hz}$ had high variability and were not considered in the average. By means of comparison, the time delay that fits the phase lag at a whisking frequency of $f_{0}=10 \mathrm{~Hz}$, i.e., $\tau=\phi / 2 \pi f_{0}=350 \mathrm{~ms}$, leads to an unrealistic frequency dependence for the phase lag (light gray line in Fig. 7C). More generally, detailed measurements of the phase shift as a function of frequency across all animals $(n=8)$ are consistent with a constant lag, with a population average of $\phi=(0.83 \pm 0.08) \cdot \pi$ radians (Fig. 8).

Previous work showed that animals can whisk after transection of the infraorbital branch of the trigeminal nerve (IoN) (Franchi 2001; Gao et al. 2001; Welker 1964). We take advantage of this finding to ask if the phase lag between the intrinsic and extrinsic muscle groups can be controlled by sensory feedback, even if past results imply that activation of at least the intrinsic muscles is independent of sensory feed-

TABLE 1. Slope of phase lag vs. whisking frequency, $d \phi / d f$, calculated for data in the range of 5-12 Hz

\begin{tabular}{ccc}
\hline \hline & \multicolumn{2}{c}{ Slope (radians/Hz) $($ mean $\pm \mathrm{SD})$} \\
\cline { 2 - 3 } Animal & Intact & Transected \\
\hline 1 & $-0.16 \pm 0.03$ & $0.10 \pm 0.05$ \\
2 & $0.12 \pm 0.03$ & $0.10 \pm 0.03$ \\
3 & $0.02 \pm 0.04^{*}$ & $0.03 \pm 0.07^{*}$ \\
4 & $-0.01 \pm 0.02^{*}$ & $0.05 \pm 0.02$ \\
6 & $0.03 \pm 0.01$ & $(0.03 \pm 0.01) \dagger$ \\
7 & $-0.04 \pm 0.01$ & $(-0.04 \pm 0.01) \dagger$ \\
8 & $0.04 \pm 0.04^{*}$ & \\
9 & $0.01 \pm 0.02^{*}$ & \\
Population average & & $0.07 \pm 0.05^{*}$ \\
(mean \pm SD) & $0.00 \pm 0.03^{*}$ & $\mathrm{C}$ \\
\hline
\end{tabular}

* Null hypothesis of 0 slope is satisfied at the $95 \%$ limit. $\dagger$ Control experiments with "sham" transections. 
back. Thus we repeated the measurements on the spectral phase lags between the intrinsic and extrinsic $\nabla$ EMGs after transection of the IoN. The lags are seen to persist and remain independent of frequency (Fig. 7, $E$ and $F$ ). The result as a function of all whisking episodes ( $n=603,1$-s bouts) shows the distributions were largely unchanged as a function of frequency (cf. Fig. 7, $G$ and $H$, with $C$ and $D$ ).

Bilateral transection of the IoN did not appear to lead to a change from a frequency-independent to a frequency-dependent phase lag (Table 1), and does not lead to a significant change in the average phase lag across animals (Fig. 8). This data implies that the phase-locked activation of the intrinsic and extrinsic muscles are not dependent on sensory feedback.

\section{Spectral properties of whisking: frequency distribution}

An outstanding observation of whisking in our trained animals is that they tended to whisk robustly at a single frequency within a bout and then changed that frequency between bouts (Fig. 6) (see also Fig. 3 in O'Connor et al. 2002). We quantified this behavior in terms of the spectral power in the intrinsic $\nabla \mathrm{EMG}$ across bouts (Fig. 9). On the basis of a single bout, we observed that the spectral power for whisking was very sharp and is thus well characterized by a center frequency, denoted $\mathrm{f}_{0}$, and a spectral width, $\Delta \mathrm{f}_{0}$, characterized by the half-width at half-maximum
(Fig. 9A, inset). We consider first the case of one animal for which we selected out bouts of $4 \mathrm{~s}$ of continuous whisking. The spectral width for over $50 \%$ of the bouts was within the Rayleigh frequency, or minimum resolvable bandwidth, of $f_{\mathrm{R}}=(4 \mathrm{~s})^{-1}=$ $0.25 \mathrm{~Hz}$ (gray arrow; Fig. $9 A$ ), i.e., $\Delta f_{0} \leq f_{\mathrm{R}}$.

Although the spectral width of the whisking response was sharp on a single trial basis, there was considerable variability in the choice of frequency, $f_{0}$, between bouts. The histogram of frequencies, expressed in terms of the probability density function (PDF), ranged from 6 to $25 \mathrm{~Hz}$ for data from this animal (Fig. 9B). The PDF has an absolute maximum with a peak whisking frequency of $f_{o}$ approximately $10 \mathrm{~Hz}$ and a half-width at half-maximum, denoted $W$, of $W=1.3 \mathrm{~Hz}$. Further, there is a second, weak maximum at the higher whisking frequency of $15 \mathrm{~Hz}$. The distribution of whisking frequencies is consistent with the description of whisking in terms of a dominant, low-frequency exploratory mode and an infrequent, high-frequency foveal mode (Fig. 9B). The distribution of frequencies is large compared with the bandwidth of whisking for a single bout, i.e., $W$ approximately $5 \cdot \Delta f_{0}$ (cf. Fig. $9, A$ and $B$ ).

In principle, the narrow spectral response observed during exploratory whisking can result either from sensory feedback that acts on an otherwise irregular oscillator or as a consequence of an accurate autonomous pattern generator. To distinguish these possibilities, we repeated the measurements on
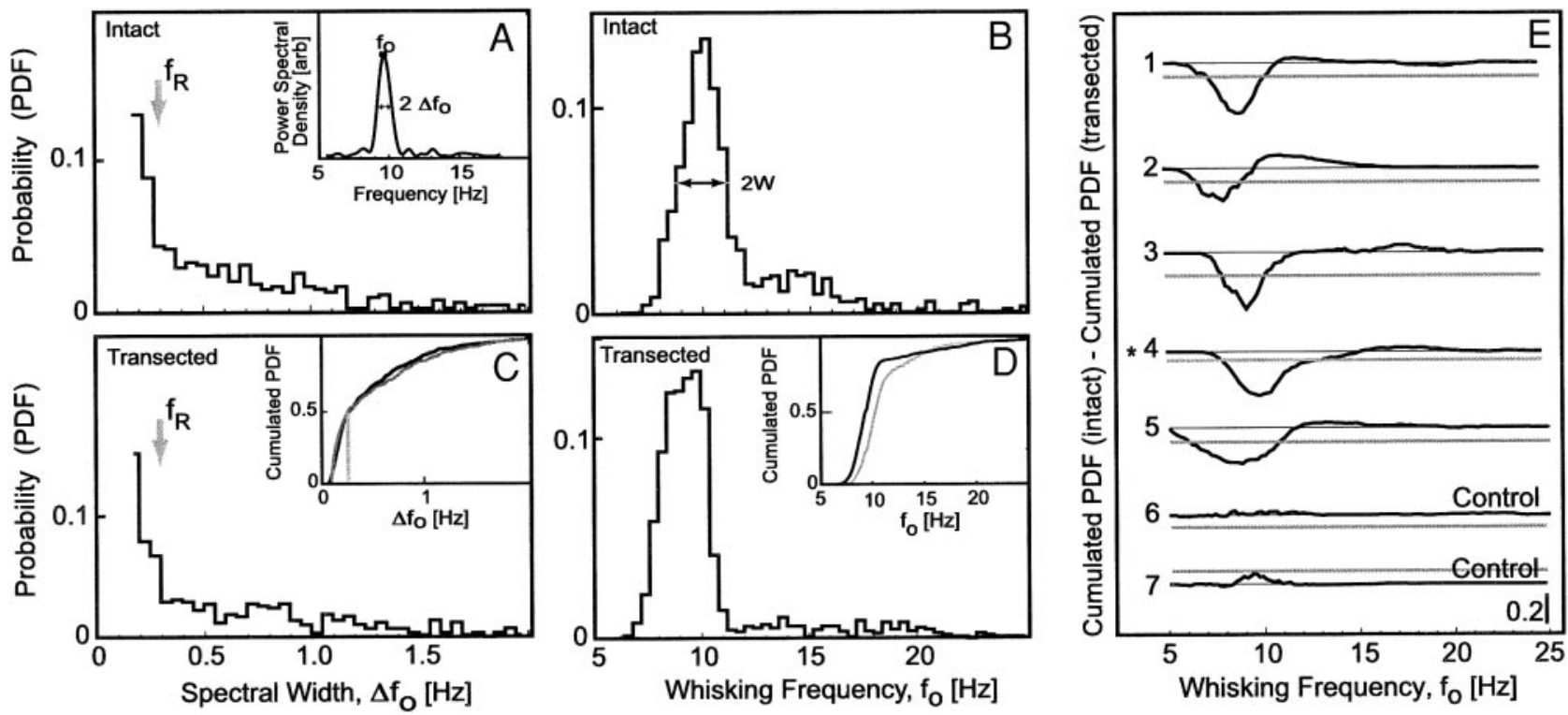

FIG. 9. The distribution of whisking spectral properties across bouts. A: probability distribution function (PDF) for the spectral bandwidth in a representative animal (4 in $E$ ) with the nervous system intact ("intact", $n=554,4$-s bouts). The measure of the bandwidth, $\Delta f_{0}$, is defined in the inset. The bin size is $0.05 \mathrm{~Hz}$. The distribution peaks within the theoretical limit, or Rayleigh frequency, of $f_{\mathrm{R}}=0.25 \mathrm{~Hz}$. Inset: spectral power density for 1 bout. The peak of the spectrum is denoted $f_{0}$. The width is defined in terms of the half-width at half-maximal amplitude. $B$ : PDF for the whisking frequency with the nervous system intact ("intact", $n=778,1$-s bouts); the bin size is $0.4 \mathrm{~Hz}$. The measure of the center frequency is defined in the inset in $A$. The distribution is bimodal with a global maximum at a frequency of $f_{0}=10.6 \mathrm{~Hz}$. There were no events below $f_{0}=6 \mathrm{~Hz}$. The width of the PDF, denoted $2 W$, is characterized by the full width at half-maximal amplitude of the dominant, low-frequency peak. $C$ : PDF for the spectral bandwidth after transection of the IoN ( $n=579,4$-s bouts). Inset: cumulative, defined as the integrated and normalized PDF, for the "intact" (gray line) vs. "transected" (black line) cases. The difference between the cumulatives, and thus the original PDFs, is not statistically significant at the $95 \%$ rejection limit by the Kolmogorov-Smirnov test. The vertical gray line corresponds to $f_{\mathrm{R}}$. $D$ : PDF for the whisking frequency after transection of the IoN ("transected", $n=1,132,1$-s bouts). This distribution has an absolute maximum at $f_{0}=9.6 \mathrm{~Hz}$. Inset: cumulatives for the "intact" (gray line) vs. "transected" (black line) cases. The difference between the cumulatives was statistically significance. E: summary of the results for 7 animals. The top 5 traces show the difference of the cumulate PDFs of whisking frequencies for the "intact" vs. "transected" cases. The shift to a lower mean frequency after transection of the IoN is seen to be statistically significant at the $95 \%$ rejection limit (gray line) in all 5 cases. The bottom 2 traces, marked "control," show the difference of the cumulated PDFs for 2 animals before and after a sham transection surgery. The frequency for both control cases is unchanged at the $95 \%$ confidence limit. 
TABLE 2. Center frequency and full bandwidth of the probability distribution function for whisking

\begin{tabular}{|c|c|c|c|c|}
\hline \multirow[b]{2}{*}{ Animal } & \multicolumn{2}{|c|}{ Center Frequency $(\mathrm{Hz})$} & \multicolumn{2}{|c|}{$\begin{array}{l}\text { Full Bandwidth, } 2 \mathrm{~W} \\
\qquad(\mathrm{~Hz})\end{array}$} \\
\hline & Intact & Transected & Intact & Transected \\
\hline 1 & 9.0 & 8.0 & 2.4 & 2.5 \\
\hline 2 & 8.2 & 7.9 & 2.6 & 2.9 \\
\hline 3 & 9.5 & & 2.4 & \\
\hline 4 & 9.8 & 8.5 & 1.5 & 2.0 \\
\hline 5 & 10.0 & 9.3 & 2.5 & 2.5 \\
\hline 6 & 9.0 & 8.1 & 3.4 & 3.0 \\
\hline 7 & 9.1 & $(9.5)^{*}$ & 3.0 & $(2.5)^{*}$ \\
\hline 8 & 9.2 & $(9.5)^{*}$ & 2.4 & $(1.9)^{*}$ \\
\hline 9 & 8.8 & & 1.9 & \\
\hline $\begin{array}{l}\text { Population average } \\
\quad(\text { mean } \pm \text { SD })\end{array}$ & $9.2 \pm 0.5$ & $8.4 \pm 0.6$ & $2.5 \pm 0.6$ & $2.6 \pm 0.4$ \\
\hline
\end{tabular}

* Control experiments for which surgery with "sham" transections were performed.

the spectral properties of whisking after transection of the IoN. We observed that the spectral width of individual bouts was unchanged (Fig. 9C), and that the distribution of the spectral widths after the transection was statistically indistinguishable from that in the intact animal (Kolmogorov-Smirnoff 2-sample test with $95 \%$ confidence interval; Fig. $9 C$, inset). In contrast, there was a small but highly significant shift (Fig. 9D, inset) in the histogram of whisking frequencies toward lower frequencies (Fig. 9D), although the width of the low-frequency peak in the PDF of whisking frequencies was unchanged (cf. $2 W$ in Fig. 9, $B$ and $D$ ). In summary, the spectral purity of individual bouts of whisking does not depend on sensory feedback.

The spectral changes on transection of the IoN were examined over a population of animals $(n=5)$. In all cases, we observed no significant change in the spectral width, $\Delta f_{\mathrm{o}}$, of the individual bouts. Nor did we observe any change in the width in the distribution of whisking frequencies for the dominant, low frequency peak, $2 W$ (Table 2). However, we did observe a significant decrease in the peak value of the distribution of whisking frequencies after the transection of the nerve. This decrease was found in all animals and is shown in terms of the difference between the cumulated PDFs for the intact versus the transected cases (Fig. $9 E$ and Table 2). It occurred mostly for the whisking frequencies below $f_{0}$ approximately $15 \mathrm{~Hz}$, so that exploratory whisking frequencies are significantly affected by the transection. Last, there was no significant change in whisking frequency after sham surgeries $(n=2)$, in which the IoN was exposed, as in the transected cases, but was not cut ("control" in Fig. 9E).

\section{Spectral properties of whisking: amplitude distribution}

We return to the observation that the extrinsic muscles are rhythmically active during exploratory whisking but are largely inactive during foveal whisking (Figs. 6, 4, $P$ and $Q$, and $5 Q$ ). We quantified this finding across animals ( 4 animals and $n=$ $2,229,1$-s bouts) in terms of the value of the square root of the spectral power density at the center frequency of whisking (Fig. 9A, inset); this quantity is proportional to the amplitude of the rhythmic vibrissa motion. It was determined across all bouts for both the intrinsic and extrinsic $\nabla$ EMG. Further, as a means to compare data across animals, as well as across the same animal on different days, we normalized the spectral power by the maximum observed value for a given animal and a given session of measurements.

Scatter plots of our results, where each point represents the average amplitude in a 1-s period of whisking, contain a preponderance of values at low frequency, i.e., between 6 and $12 \mathrm{~Hz}(85 \%$ of all events; Fig. 9B). This distribution corresponds to the prevalence of exploratory over foveal whisking. Nonetheless, the normalized value of the amplitude for the intrinsic $\nabla \mathrm{EMG}$ is essentially constant $\leq 17 \mathrm{~Hz}$ and that the amplitude is still substantial $\leq 25 \mathrm{~Hz}$ (solid line in Fig. 10A). In
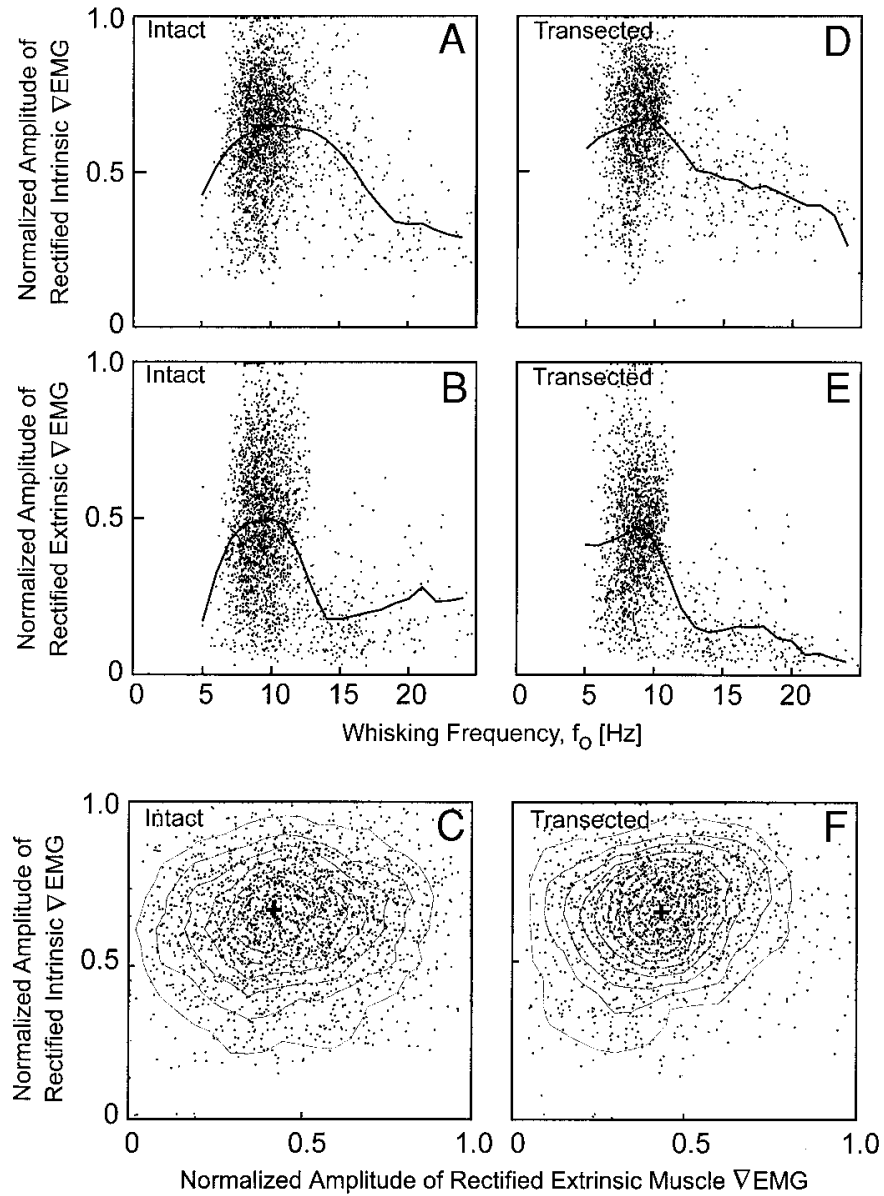

FIG. 10. The distribution of amplitudes, calculated as the square root of spectral power at the whisking frequency, in the intrinsic vs. extrinsic muscles. The power was normalized by the maximum value obtained for the complete set of trials, typically 100 bouts that were $1 \mathrm{~s}$ in duration, in a given recording session. $A$ : amplitude of the rectified $\nabla \mathrm{EMG}$ for the intrinsic muscles as a function of frequency for animals with an intact nervous system $(n=2,229$ bouts across 4 animals). The solid curve is a fit to the average amplitude as a function of frequency. Note that the normalized amplitude for this muscle group falls-off at high frequencies. $B$ : amplitude of the $\nabla \mathrm{EMG}$ for the extrinsic muscles as a function of frequency (all conditions as in $A$ ). Note that the normalized amplitude for this muscle group falls off sharply above $10 \mathrm{~Hz}$. $C$ : amplitude of the $\nabla \mathrm{EMG}$ for the intrinsic muscles vs. that for the extrinsic muscles. The solid curves are contours of constant normalized amplitude drawn at intervals of 0.12 with the 1st contour at 0.12 . Note the relative independence of the activity of the intrinsic vs. extrinsic $\nabla$ EMG. $D-F$ : amplitude of the $\nabla$ EMG for the intrinsic and extrinsic muscles after transection of the IoN ("transected") as a function of frequency ( $n=$ 1,706 bouts across 4 animals); the panels are arranged analogously to those for the "intact" case $(A-C)$. Note that the sole effect of transection is that the spread of the amplitude for the extrinsic $\nabla \mathrm{EMG}$ is narrower for the "transected" case than for the "intact" case (cf. $C$ and $F$; the cross is a fiducial to aid in comparing the responses). 
contrast, the normalized value of the amplitude for the extrinsic $\nabla \mathrm{EMG}$ is seen to fall to near zero for whisking frequencies above $12 \mathrm{~Hz}$ (Fig. 10B). These data demonstrate that lowfrequency, exploratory whisking is accompanied by activity of the extrinsic as well as the intrinsic muscles as a general phenomenon across animals. High-frequency, foveal whisking is driven almost exclusively by the intrinsic muscles. This difference in muscular activation provides a distinction among the patterns of whisking observed in our behavior task.

As a means to address the possible correlation between the extent of activation of the extrinsic versus intrinsic muscles across bouts (Fig. 10C), we constructed a scatter plot of the amplitude in the two $\nabla$ EMG signals for exploratory whisking. The essential conclusion is that the typical activation of the intrinsic muscles is 0.6 times of its maximum value, while the concurrent activation of the extrinsic muscles is only 0.4 times the maximum value $(+$ in Fig. 10C). Furthermore, there is no significant correlation between the activation of the two muscle groups. Thus although the time dependence of the two muscle groups is locked, their relative amplitudes across whisking bouts is independent.

To address the possibility that the distribution of whisking amplitudes depends on sensory feedback, we repeated the measurements on the distribution of spectral power in the intrinsic and extrinsic muscle $\nabla$ EMG after transection of the IoN ( $n=1,706,1$-s bouts). We observed that the distribution of the intrinsic $\nabla \mathrm{EMG}$ amplitude was largely unchanged as a function of frequency (Fig. 10D), while the distribution of the extrinsic $\nabla$ EMG amplitude slightly narrowed (Fig. 10E). With regard to the issue of correlated activation of the follicular and extrinsic muscles during exploratory whisking, we observed that transection of the IoN lead to a small but significant correlation between the activation of the two muscle groups (Fig. 10F; correlation coefficient of 0.14).

\section{I S C U S S I O N}

We have addressed the activity of two sets of musculature whose contraction would be expected to control the position of the vibrissae (Fig. 3). For the relatively large amplitude sweeps associated with exploratory whisking, both the intrinsic and the extrinsic muscle groups are activated in an antiphasic pattern (Figs. 4, 6, 7, and 8). The intrinsic muscles protract the vibrissae while the extrinsic muscles are correlated with retraction (Fig. 4). In terms of the known anatomy, protraction is accomplished through the exertion of a torque on the follicle while retraction is accomplished by movement of the attachment point of a follicle (Fig. 1D). The amplitude of the activity of the two muscle groups, as inferred from the spectral power in the respective $\nabla$ EMGs, are essentially uncorrelated between whisking bouts (Fig. 10). In contrast, the peak of the activation of the two muscle groups is approximately anti-phasic (Figs. 4, 6,7 , and 8) and independent of frequency over the 5- to $15-\mathrm{Hz}$ range of exploratory whisking (Figs. 7 and 8). This antiphasic pattern of muscle activation persisted after transection of the trigeminal nerve (Figs. 7 and 8).

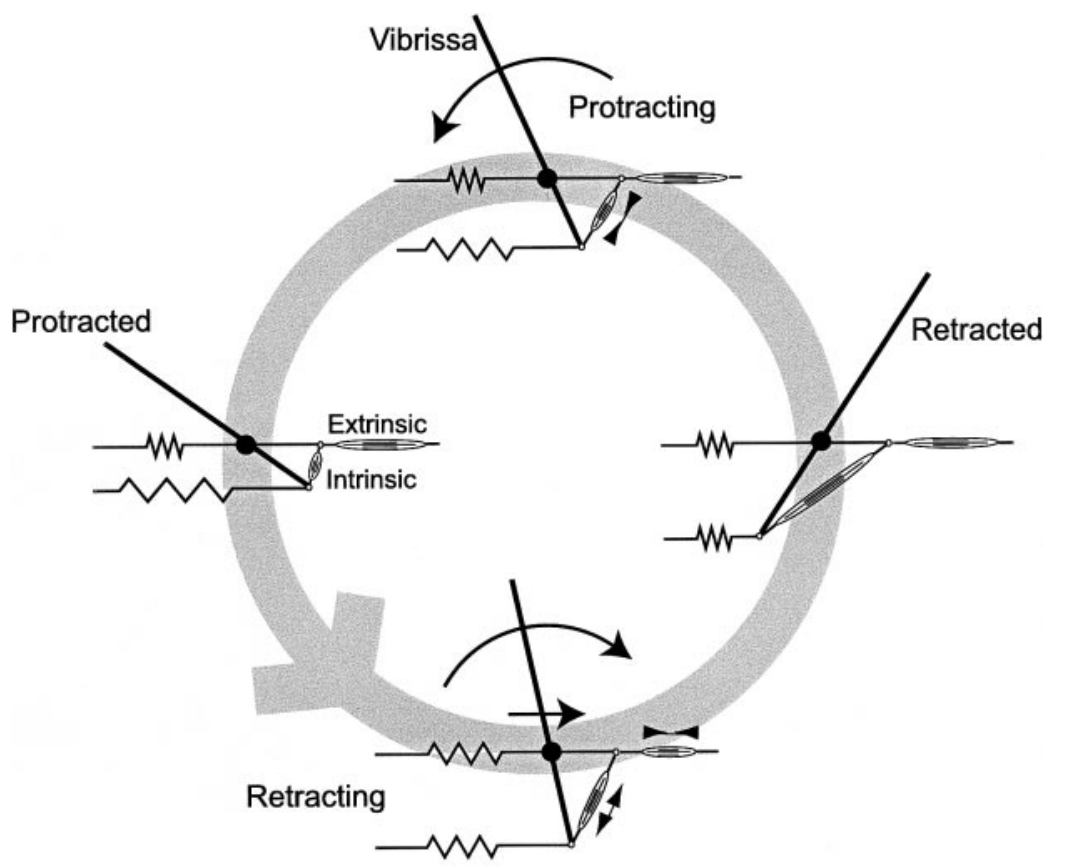

FIG. 11. Mechanical model that relates cyclic vibrissa movements to the alternating intrinsic and extrinsic muscles during exploratory whisking. The components are those deduced from Dörfl's (1982) anatomical studies (Fig. 1D). Note that retraction involved a change on the pivot point as well as the angle. The relation between mechanical stages and the intrinsic and extrinsic $\nabla \mathrm{EMG}$ is meant only to be approximate.

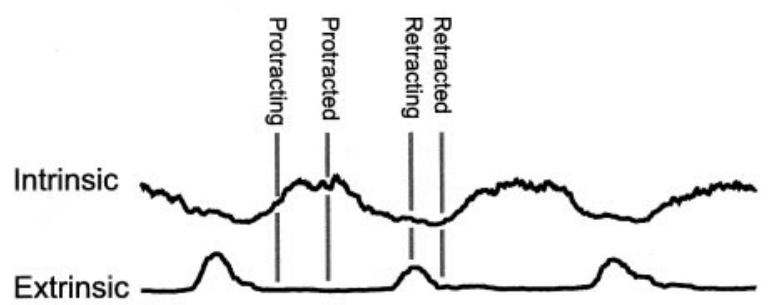

Time 
In contrast to the large amplitude sweeps associated with exploration, a second pattern of whisking, denoted foveal whisking, occurred when the animal perched and the vibrissae were thrust forward and stretched toward an object (Fig. 5). This pattern consists of relatively low-amplitude whisks in the frequency range of 15-25 Hz (Figs. 9 and 10). Protraction of the vibrissae is driven by the intrinsic muscles (Fig. 1B), while retraction is largely passive as the extrinsic muscles are essentially inactive (Figs. 6 and 10).

\section{Active retraction and the mechanics of whisking}

Based on the consideration of anatomical studies, Dorfl (1982) proposed that the fibrous connective tissue at the base of the follicle could function as both a damper and a spring to allow the vibrissae to passively retract after a protraction (plate in Fig. $1 B$ and $D$ ). This led to the hypothesis that the retraction of the vibrissae is a passive process, i.e., that retraction occurs without the involvement of muscular activation. Here we confirm this for the case of foveal whisking (Figs. 5, 6, and 10). In addition, we now show that during exploratory whisking, retraction is an active process (Figs. 4, 6-8, and 10).

From the point of view of mechanics, there are two degrees of freedom that describe the motion. These correspond to the angle of the vibrissae relative to the plane of the mystacial pad and to the position of the apex of the follicle, which serves as a pivot point (Fig. 1D). Under steady-state conditions, both degrees of freedom are phase-locked to each other throughout the whisking cycle (Figs. 7 and 8), as modeled schematically in Fig. 11. Contraction of the intrinsic muscles results in a forward rotation of the vibrissae with respect to the pivot point, i.e., protraction. In contrast, contraction of the extrinsic muscles produces a force that moves the pivot point backward and leads to counter rotation of the vibrissae, i.e., retraction. Our mechanical model anticipates an alternating activation of the intrinsic and extrinsic muscles (Fig. 11), as was observed for exploratory whisking (Figs. 6, 4, 8, and 10). Our mechanical model also provides a means to understand the passive nature of retraction observed for foveal whisking. The extrinsic muscles cannot exert an appreciable torque when the vibrissae are thrust forward, consistent with their relative silence during foveal whisking (Figs. 5, 6, and 10B).

\section{Spectral purity of whisking}

Rats are observed to whisk in the frequency range of 5-15 $\mathrm{Hz}$ when they explore their immediate environment in search of an object or food (Figs. 4, 6, 9, and 10). The frequencies are

\section{A Single oscillator with sensory feedback}
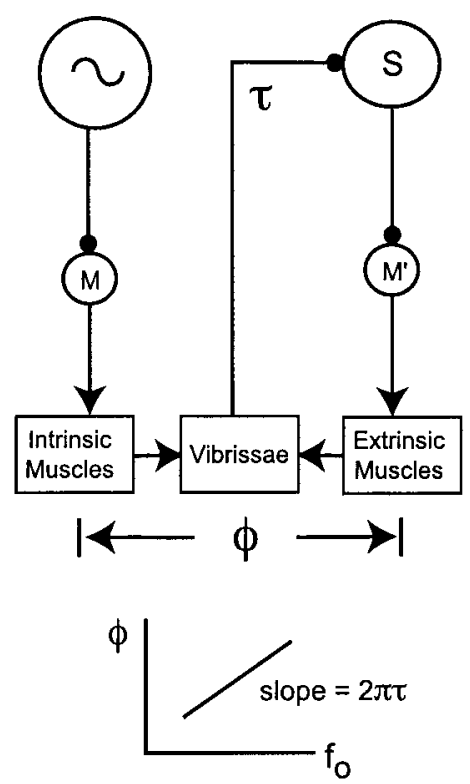
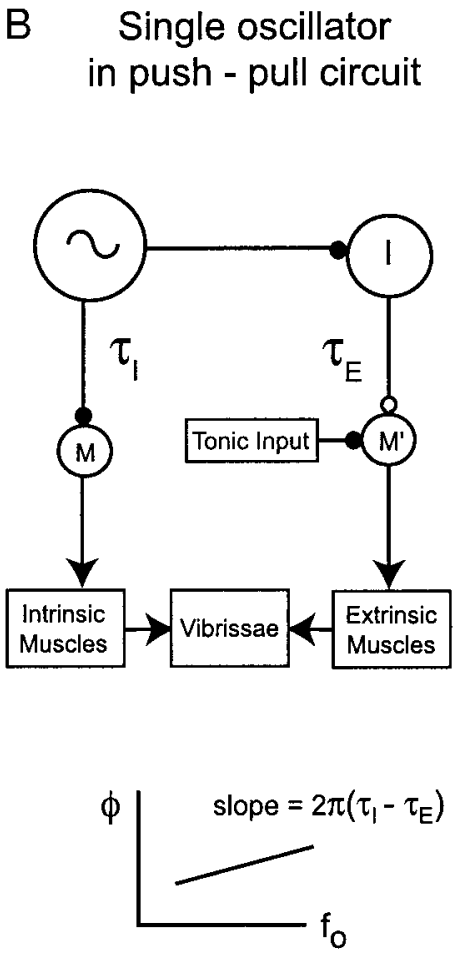
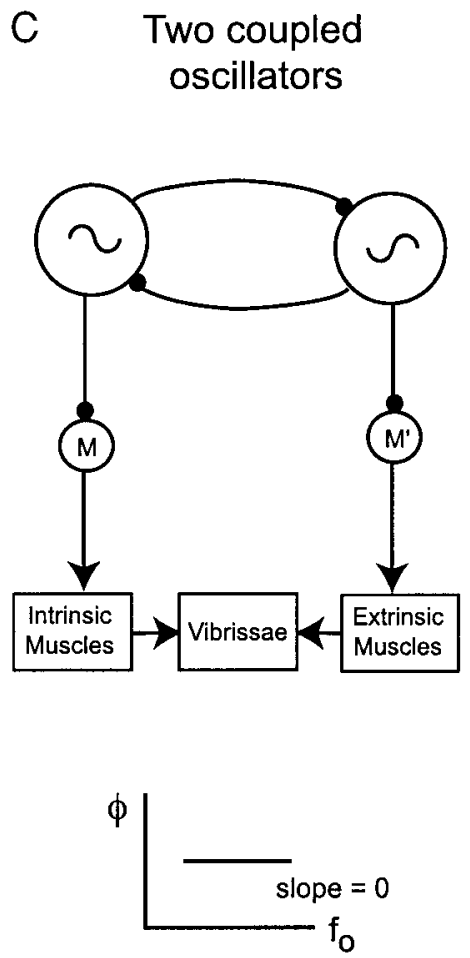

FIG. 12. Minimalist neuronal circuits for the generation of the observed alternating muscle activity between the intrinsic and extrinsic muscles during exploratory whisking. A: a serial scheme. A single oscillator drives motoneurons (M) that activates the intrinsic muscles. This motion is detected by the trigeminal sensory neurons (S) and used to drive a different set of motoneurons $\left(\mathrm{M}^{\prime}\right)$ that activate the extrinsic muscles. The serial nature of the circuit leads to a constant time lag, denoted $\tau$, between activation of the 2 muscle groups, so that a plot of the phase lag vs. whisking frequency is linear, with a slope of $\Delta \phi / \Delta f=2 \pi \tau$. $B$ : a parallel scheme with a single oscillator. An inhibitory interneuron (I) is used to generate a phase difference near $\pi$ radians for the drive to the motoneurons for the intrinsic vs. extrinsic muscles. However, the asymmetry in the circuit introduces a relative time delay between activation of the intrinsic muscles, with delay $\tau_{\mathrm{I}}$, vs. activation of the extrinsic muscles, with delay $\tau_{\mathrm{E}}$, such that the phase lag has a small slope, i.e., $\Delta \phi / \Delta f=2 \pi\left(\tau_{\mathrm{E}}-\tau_{\mathrm{I}}\right)$, as a function of whisking frequency. Note the tonic drive that biases the rhythmically inhibited motoneuron $\left(\mathrm{M}^{\prime}\right)$ into the active state. $C$ : a parallel scheme with 2 coupled oscillators that maintain a fixed phase relation. The drive to the motoneurons for the intrinsic vs. extrinsic muscles is symmetric, and thus the phase is constant, i.e., $\Delta \phi / \Delta f \approx 0$. The predictions from this model are most consistent with our observations that the phase is relatively independent of frequency (Fig. 7, $C$ and $G$ ). 
distributed with a mean center value of $9 \mathrm{~Hz}$ (Table 1), in agreement with that found in previous studies for exploratory whisking (Carvell et al. 1991; Fee et al. 1997; O'Connor et al. 2002; Welker 1964), and a full bandwidth of $2.5 \mathrm{~Hz}$ (Table 2). We further observed that the purity of whisking is very high, in the sense that the rat maintains a fixed frequency of whisking across a given bout to within the theoretical limit of spectral resolution (Fig. 9A). Thus for a 4-s bout of whisking with a frequency of $f_{0}=9 \mathrm{~Hz}$, the fractional variability across the bout is $\Delta f_{0} / f_{0}=(1 / 4 \mathrm{~s})(1 / 9 \mathrm{~Hz}) \cong 0.03$. Interesting, the frequency varies between different bouts over a much larger distribution (Table 2). These data suggest that the rat may use a frequency-based scheme for the detection of vibrissa contact during the whisk cycle (Ahissar et al. 1997; Ahissar 1998; Ahrens et al. 2002; Kleinfeld et al. 1999; see Ahissar and Kleinfeld 2002 for review). The reason for the change in frequency and amplitudes between bouts is not clear, but could constitute a means to circumvent entrainment and adaptation of neuronal oscillatory circuits.

\section{Sensory deafferentation and whisking}

Deflection of the vibrissae relative to that of the follicle, as would occur during contact of the hair with an object, is relayed via the infraorbital branch of the trigeminal nerve (Dorfl 1985; Rice and Arvidsson 1991) ("sn" in Fig. 1A) to the trigeminal nuclei. In turn, trigeminal nuclei provide input to higher brain areas and putative inhibitory feedback to the lateral facial nucleus. We show that bilateral transections of the IoN do not effect the spectral purity of whisking during exploratory whisking (cf. Fig. 9, $A$ and $C$ ). Furthermore, the transections do not compromise the phase relation between the intrinsic muscles and the extrinsic muscles (Figs. 7 and 8; Table 1). These data lend support to the notion of a highly precise central pattern generator for whisking.

With regard to the effect of bilateral transections of the IoN on the distribution of whisking frequencies, an early report by Welker (1964) showed a decrement in the average center frequency of whisking from 8 to $6 \mathrm{~Hz}$ following deafferentation of the mystacial pads. These experiments involved the use of free ranging animals, similar to those in the present study. In qualitative support of this result, we observed a statistically significant decrement in whisking frequency on bilateral transection of the IoN (cf. Fig. 9, $B$ and $D$ ). This decrement occurred across all animals with an average magnitude of about $1 \mathrm{~Hz}$ (Fig. 9C; Table 2). It is of note that under the behavioral paradigm of a head-fixed preparation and different sampling conditions, the reduction in whisking frequency is not seen under similar nerve transection (Gao et al. 2001).

\section{Candidate models for a whisking central pattern generator}

We considered generic circuits for the generation of a twophase rhythm for whisking (Fig. 11). The first circuit is a serial scheme that relies on sensory feedback (Fig. 12A). The second and third models involve complementary scheme to autonomously generate a two phase output (Fig. 12, $B$ and $C$ ). We argue that the first scheme is inconsistent with the data.

In the serial scheme, a single oscillator, or oscillatory network, drives the motoneurons that control the intrinsic muscles (Fig. 12A). This motion is sensed via the trigeminal innervation of the vibrissae and used to signal the motoneurons that drive the extrinsic muscles. The time delay between the muscle groups is thus a constant, so that the phase-lag should vary as a linear function of frequency (light gray lines in Fig. 7, $C$ and $G)$. We reject this model on two grounds. First, the alternation between muscle groups should not persist in the absence of sensory feedback. Yet this alternation is unaffected by transection of the IoN (Figs. 7, $E, F$, and $G$, and 8). Second, even if a yet undiscovered sensory pathway exists along the facial nerve, this model implies that the alternation in phase between the two muscle groups should depend on the frequency of whisking. Yet the observed alternation is independent of frequency (Figs. 7 and 8; Table 1).

The second model used a single oscillator in a push-pull arrangement (Fig. 12B). The oscillator drives the motoneurons for the set of intrinsic muscles either directly, or through excitatory interneurons, and drives the set of motoneurons for the extrinsic muscles through inhibitory interneurons. This can lead to the observed antiphasic relation between the two muscle groups (Fig. 4Q). Inhibitory interneurons that contact the facial motoneurons have been reported ( $\mathrm{Li}$ et al. 1997). An additional tonic input that is required to bias the motoneurons that are driven by inhibitory input could be provided by serotonergic projections (Dolphin and Greengard 1981; McCall and Aghajanian 1979). The push-pull model does not depend on sensory feedback. However, the phase difference between the activation of the two muscles groups depends on the relative time-lag between the pathway to the intrinsic muscles, denoted $\tau_{\mathrm{I}}$, and the pathway to the extrinsic muscles, denoted $\tau_{\mathrm{E}}$, i.e., $\phi=2 \pi f_{\text {whisk }}\left(\tau_{\mathrm{E}}-\tau_{\mathrm{I}}\right)$. To estimate the feasibility of this model, we equate the slope $d \phi / d f=2 \pi\left(\tau_{\mathrm{E}}-\tau_{\mathrm{I}}\right)$ with the $\mathrm{SD}$ of \pm 0.03 radians $/ \mathrm{Hz}$ for the measured value of $d \phi / d f$ (Table 1). This implies that $\tau_{\mathrm{E}}-\tau_{\mathrm{I}}<5 \mathrm{~ms}$, a time difference that is small, although not inconsistent with the jitter in the activation of interneurons.

The third model consisted of two coupled oscillators (Fig. $12 C$ ). This circuit generates a constant phase relation between the two oscillators through a set of mutual reciprocal connections (Hansel et al. 1993; von der Vreeswijk et al. 1994). Candidate oscillators are likely to be located in the medulla and may involve reciprocal connections between the parvicellular or gigantocellular reticular nucleus and the lateral aspect of the facial nucleus (Fay and Norgren 1997; Hattox et al. 2001; Isokawa-Akesson and Komisaruk 1987; Mogoseanu et al. 1994). The output of the coupled oscillator model is independent of sensory feedback and the phase versus frequency relation is a constant by construction. While the behavior of both the push-pull model and the coupled oscillator model are consistent with the data, we favor the latter model based solely on it's relative simplicity and on the known stability of coupled oscillator systems (Kopell and Ermentrout 1986).

To summarize, the interpretation of our data suggest that the neuronal drive to the muscles for both exploratory and foveal whisking can be modeled in terms of a two-phase central pattern generator. This oscillator is presumably activated by high-level tonic input, similar to the case of mastication (Nakamura and Katakura 1995; Nozaki et al. 1986). Three independent signals, one that is used to set the frequency of whisking and two that are used to control the maximum amplitude of the intrinsic and the extrinsic musculature, respectively, must be specified for each whisking bout. Anatomical 
studies have identified direct and indirect projections from vibrissa motor cortex to hindbrain nuclei that are candidates to carry these signals (Hattox et al. 2001; Miyashita and Shigemi 1995; Miyashita et al. 1994). The details of this surprisingly highly tuned circuitry remain to be unraveled.

We thank F. F. Ebner and R.N.S. Sachdev for critical discussions, B. Friedman for instruction with the nerve transection, S. Hefler for assistance with the animal husbandry, G. A. White for programming of the video camera interface, and B. Friedman and H. P. Zeigler for comments on an early version of this work.

This study was supported by the National Institute of Mental Health and the Whitehall Foundation.

\section{REFERENCES}

Ahissar E. Temporal-code to rate-code conversion by neuronal phase-locked loops. Neural Comput 10: 597-650, 1998.

Ahissar E, Haidarliu S, and Zackenhouse M. Decoding temporally encoded sensory input by cortical oscillators and thalamic phase comparators. Proc Natl Acad Sci USA 94: 11633-11638, 1997.

Ahissar E and Kleinfeld D. Closed loop neuronal computations: focus on vibrissa somatosensation in rat. Cereb Cortex In press.

Ahrens KF, Levine H, Suhl H, and Kleinfeld D. Spectral mixing of rhythmic neuronal signals in sensory cortex. Proc Natl Acad Sci USA 99: 15176-15181, 2002

Berg RW and Kleinfeld D. Extrinsic mystacial muscles have oscillatory activity out of phase with intrinsic follicular muscles during rhythmic whisking by rat. Soc Neurosci Abstr 289: 18, 2001.

Brecht M, Preilowski B, and Merzenich MM. Functional architecture of the mystacial vibrissae. Behav Brain Res 84: 81-97, 1997.

Buzsaki G, Bickford RG, Ponomareff G, Thal LJ, Mandel R, and Gage FH. Nucleus basalis and thalamic control of neocortical activity in the freely moving rat. J Neurosci 8: 4007-4026, 1988.

Cacciatore TW, Brodfueher PD, Gonzalez JE, Jiang T, Adams SR, Tsien RY, Kristan WB Jr, and Kleinfeld D. Identification of neural circuits by imaging coherent electrical activity with FRET-based dyes. Neuron 23: 449-459, 1999.

Carvell GE and Simons DJ. Biometric analyses of vibrissal tactile discrimination in the rat. J Neurosci 10: 2638-2648, 1990.

Carvell GE and Simons DJ. Task-and subject-related differences in sensorimotor behavior during active touch. Somat Motor Res 12: 1-9, 1995.

Carvell GE, Simons DJ, Lichtenstein SH, and Bryant P. Electromyographic activity of mystacial pad musculature during whisking behavior in the rat. Somat Motor Res 8: 159-164, 1991.

Darian-Smith I. The sense of touch: performance and peripheral neural processes. In: The Nervous System, edited by Brookhart JM and Mountcastle VB. Bethesda, MD: American Physiological Society, 1984, p. 739-788.

Dolphin AC and Greengard P. Serotonin stimulates phosphorylation of protein I in the facial motor nucleus of rat brain. Nature 289: 76-79, 1981.

Dorfl J. The musculature of the mystacial vibrissae of the white mouse. J Anat 135: 147-154, 1982.

Dorfl J. The innervation of the mystacial region of the white mouse. A topographical study. J Anat 142: 173-184, 1985.

Fay RA and Norgren R. Identification of rat brainstem multisynaptic connections to the oral motor nuclei in the rat using pseudorabies virus. II Facial muscle motor systems. Brain Res Rev 25: 276-290, 1997.

Fee MS, Mitra PP, and Kleinfeld D. Central versus peripheral determinates of patterned spike activity in rat vibrissa cortex during whisking. $J$ Neurophysiol 78: 1144-1149, 1997.

Franchi G. Persistence of vibrissal motor representation following vibrissal pad deafferentation in adult rats. Exp Brain Res 137: 180-189, 2001.

Freeman WJ and Baird B. Relation of olfactory EEG to behavior: spatial analysis. Behav Neurosci 101: 393-408, 1987.

Gao P, Bermejo R, and Zeigler HP. Vibrissa deaffentation and rodent whisking patterns: behavioral evidence for a central pattern generator. J Neurosci 21: 5374-5380, 2001.

Gioanni Y and Lamarche M. A reappraisal of rat motor cortex organization by intracortical microstimulation. Brain Res 344: 49-61, 1985.

Guic-Robles E, Valdivieso C, and Guajardo G. Rats can learn a roughness discrimination using only their vibrissal system. Behav Brain Res 31: 285-289, 1989.
Hansel D, Mato G, and Meunier C. Phase dynamics for weakly coupled Hodgkin-Huxley neurons. Eur Physiol Lett 23: 367-372, 1993.

Hattox AM, Priest CA, and Keller A. Functional circuitry involved in the regulation of whisker movements. J Comp Neurol 442: 266-276, 2001.

Isokawa-Akesson M and Komisaruk BR. Difference in projections to the lateral and medial facial nucleus: anatomically separate pathways for rhythmical vibrissa movement in rats. Exp Brain Res 65: 385-398, 1987.

Kleinfeld D, Berg RW, and O'Connor SM. Anatomical loops and their relation to electrical dynamics in relation to whisking by rat. Somat Motor Res 16: 69-88, 1999.

Koehl MAR, Koseff JR, Crimaldi JP, McCay MG, Cooper T, Wiley MB, and Moore PA. Lobster sniffing: anrennule design and hydrodynamic filtering of information in an odor plume. Science 294: 1948-1950, 2001.

Kopell N and Ermentrout GB. Symmetry and phaselocking in chains of weakly coupled oscillators. Commun Pure Appl Math 39: 623-660, 1986.

Li YG, Takada M, Keneko T, and Mizuno N. Distribution of GABAergic and glycinergic premotor neurons projecting to the facial and hypoglossal nuclei in the rat. J Comp Neurol 378: 283-294, 1997.

McCall RB and Aghajanian GK. Serotonergic facilitation of facial motoneuron excitation. Brain Res 169: 11-27, 1979.

Miyashita E, Keller A, and Asanuma $\mathbf{H}$. Input-output organization of the rat vibrissal motor cortex. Exp Brain Res 99: 223-232, 1994.

Miyashita E and Shigemi M. The superior colliculus relays signals descending from the vibrissal motor cortex to the facial nerve nucleus in the rat. Neurosci Lett 195: 69-71, 1995.

Mogoseanu D, Smith AD, and Bolam JP. Monosynaptic innervation of facial motoneurons by neurones of the parvicellular reticular formation. Exp Brain Res 101: 427-438, 1994.

Nakamura $\mathbf{Y}$ and Katakura N. Generation of masticatory rhythm in the brainstem. Neurosci Res 23: 1-19, 1995.

National Institutes of Health. Guide for the Care and Use of Laboratory Animals, Bethesda, MD: National Institutes of Health, 1985.

Nicolelis MAL, Baccala LA, Lin RCS, and Chapin JK. Sensorimotor encoding by synchronous neural ensemble activity at multiple levels of the somatosensory system. Science 268: 1353-1358, 1995.

Nozaki S, Iriki A, and Nakamura Y. Location of central rhythm generator involved in cortically induced rhythmical masticatory jaw-opening movement in the guinea pig. $J$ Neurophysiol 55: 806-825, 1986.

O'Connor SM, Berg RW, and Kleinfeld D. Coherent electrical activity along vibrissa sensorimotor loops during free whisking in rat. J Neurophysiol 87: 2137-2148, 2002.

Rashbass C. The relationship between saccadic and smooth tracking eye movements. J Physiol (Lond) 159: 326-338, 1961.

Rice FL and Arvidsson J. Central projections of primary sensory neurons innervating different parts of the vibrissae follicles and intervibrissal skin on the mystacial pad of the rat. J Comp Neurol 309: 1-16, 1991.

Rice FL, Fundin BT, Pfaller K, and Arvidsson J. The innervation of the mystacial pad in the adult rat studied by anterograde transport of HRP conjugates. Exp Brain Res 99: 233-246, 1994.

Sachdev RN, Sato T, and Ebner FF. Divergent movement of adjacent whiskers. J Neurophysiol 87: 1440-1448, 2002.

Sanderson KJ, Welker W, and Shambes GM. Reevaluation of motor cortex and of sensorimotor overlap in cerebral cortex of albino rats. Brain Res 292: 251-260, 1984.

Semba K and Komisaruk BR. Neural substrates of two different rhythmical vibrissal movements in the rat. Neuroscience 12: 761-774, 1984.

Simons DJ and Carvell GE. Abnormal tactile experience early in life disrupts active touch. J Neurosci 16: 2750-2757, 1996.

Thomson DJ. Spectral estimation and harmonic analysis. Proc IEEE 70: 1055-1096, 1982.

Thomson DJ and Chave AD. Jackknifed error estimates for spectra, coherences, and transfer functions. In: Advances in Spectrum Analysis and Array Processing, edited by Shykin S. Upper Saddle River, NJ: Prentice Hall, 1991, p. $58-113$.

Vincent SB. The function of the vibrissae in the behavior of the white rat. Behav Monographs 1: 7-81, 1912.

von der Vreeswijk C, Abbott LF, and Ermentrout GB. When inhibition not excitation synchronizes neural firing. J Comput Neurosci 4: 313-321, 1994.

Welker WI. Analysis of sniffing of the albino rat. Behaviour 12: 223-244, 1964

Wineski LE. Movements of the cranial vibrissae in the golden hamster (Mesocritus auratus). J Zool (Lond) 200: 261-280, 1983.

Wineski LE. Facial morphology and vibrissal movement in the golden hamster. J Morphol 183: 199-217, 1985. 